J. Zintl

Nagoya Math. J.

Vol. 196 (2009), 27-66

\title{
THE ONE-DIMENSIONAL STRATUM IN THE BOUNDARY OF THE MODULI STACK OF STABLE CURVES
}

\author{
JÖRG ZINTL
}

\begin{abstract}
It is well-known that the moduli space $\bar{M}_{g}$ of Deligne-Mumford stable curves of genus $g$ admits a stratification by the loci of stable curves with a fixed number $i$ of nodes, where $0 \leq i \leq 3 g-3$. There is an analogous stratification of the associated moduli stack $\overline{\mathcal{M}}_{g}$.

In this paper we are interested in that particular stratum of the moduli stack, which corresponds to stable curves with exactly $3 g-4$ nodes. The irreducible components of this stratum are one-dimensional substacks of $\overline{\mathcal{M}}_{g}$. We show how these substacks can be related to simpler moduli stacks of (permutation classes of) pointed stable curves. Furthermore, we use this to construct all of the components of this boundary stratum generically in a new way as explicit quotient stacks.
\end{abstract}

\section{$\S 1$. Introduction}

A stable curve $f: C \rightarrow \operatorname{Spec}(k)$ of genus $g \geq 2$ in the sense of Deligne and Mumford $[\mathrm{DM}]$ may have at most $3 g-3$ nodes. The locus of curves with exactly $i$ nodes is a subscheme $M_{g}^{(i)}$ of the moduli space $\bar{M}_{g}$, and it is of dimension $3 g-3-i$. Thus there is a stratification of the moduli space of Deligne-Mumford stable curves

$$
\bar{M}_{g}=M_{g}^{(0)} \cup \cdots \cup M_{g}^{(3 g-3)} .
$$

If the maximal number of nodes is attained by a stable curve, then this curve decomposes into $2 g-2$ irreducible components. Up to isomorphisms, there are only finitely many such curves. Therefore their locus $M_{g}^{(3 g-3)}$ is discrete in $\bar{M}_{g}$.

Our objects of study in this paper are the connected components of the subscheme $M_{g}^{(3 g-4)}$. Each connected component $D$ of this stratum can

Received March 9, 2008.

Revised February 7, 2009.

Accepted March 18, 2009.

2000 Mathematics Subject Classification: Primary 14H10; Secondary 14H37. 
be identified with a corresponding much simpler moduli space $M$. Before we make this formal in Section 3, let us briefly sketch the idea behind this approach.

1.1. Let $f_{0}: C_{0} \rightarrow \operatorname{Spec}(k)$ be a fixed stable curve of genus $g$ with $3 g-3$ nodes, and let one node $P_{1}$ of $C_{0}$ be fixed. The pair $\left(C_{0}, P_{1}\right)$ distinguishes one connected component $D$ of the stratum $M_{g}^{(3 g-4)}$, as we will explain in Section 2. Indeed, for any deformation of $C_{0}$, which preserves all nodes of $C_{0}$ except $P_{1}$, all fibres of the deformation are represented by points of $D$. Each connected component of $M_{g}^{(3 g-4)}$ can be obtained in this way.

The geometry of the boundary $\bar{M}_{g} \backslash M_{g}^{(0)}$ is well known, see for example [HM]. It is a normal crossing divisor, and the strata $M_{g}^{(i)}$ for $i \geq 1$ are cut out by intersections of its irreducible components. In particular, the closures of the one-dimensional subschemes $D$ in $\bar{M}_{g}$ are meeting each other transversally exactly in the points of $M_{g}^{(3 g-3)}$. Therefore the connected components $D$ are simultaneously the irreducible components of $M_{g}^{(3 g-4)}$. We will see below that for each $D$ its closure is a rational curve, and it is even nonsingular, compare Lemma 3.4 and 2.5.

A closed point of $D$ represents a stable curve $f: C \rightarrow \operatorname{Spec}(k)$ with exactly $3 g-4$ nodes, and at least $2 g-3$ irreducible components. Each of the irreducible components of $C$ can be considered as a pointed stable curve itself, the special points being the points of intersection with other components. There is exactly one irreducible component $C^{+}$of $C$, which has not the maximal number of nodes, when considered as a pointed stable curve. The closure of the complement of $C^{+}$in $C$ is a pointed prestable curve $C_{0}^{-}$, which is the same for all curves represented by closed points of $D$. In fact, $C_{0}^{-}$is the closure of the complement of $C_{0}^{+}$in $C_{0}$, where $C_{0}^{+}$is the union of all irreducible components of $C_{0}$ which contain the node $P_{1}$. Note that $C_{0}^{-}$needs not to be connected.

EXAmple 1.2. Consider the stable curve $C_{0}$ of genus $g=3$ as pictured below. It attains the maximal number of nodes $3 g-3=6$. Each component of $C_{0}$ is a rational curve. Let $P_{1}, \ldots, P_{6}$ be an enumeration of the nodes of $C_{0}$ as indicated. The node $P_{1}$ determines a decomposition of $C_{0}$ into two 2-pointed subcurves $C_{0}^{-}$and $C_{0}^{+}$as shown on the right hand side of the arrow in the picture. 

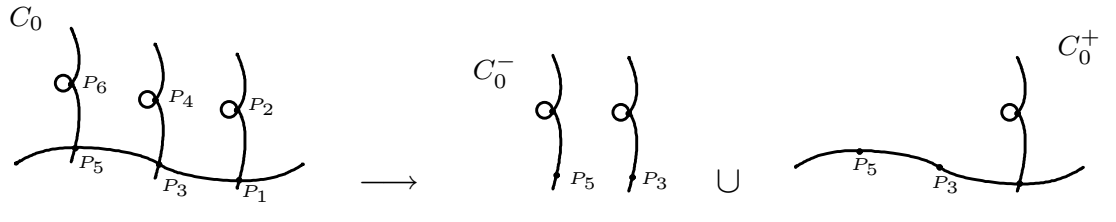

A curve $C$, obtained by a deformation of $C_{0}$ preserving all nodes except $P_{1}$, looks schematically like this.

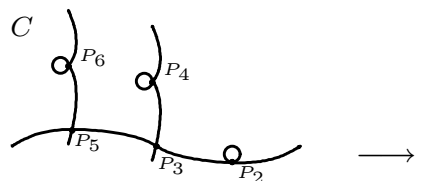

$$
\mathcal{C}_{0}^{-} \mathcal{L}_{P_{5}}
$$

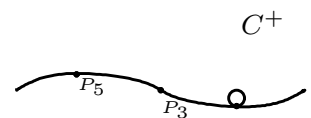

The stable curve $C$ is represented by a point in some connected component $D$, which lies inside the stratum $M_{3}^{(5)}$. It decomposes as $C=C^{+} \cup C_{0}^{-}$, as pictured above, where the subcurve $C^{+}$is a 2-pointed stable curve of genus $g^{+}=1$.

In general, if a pair $\left(C_{0}, P_{1}\right)$ is fixed, then there are numbers $g^{+} \leq g$ and $m \leq 4$, such that for any curve $C$ represented by a point of $D$ there exists a curve $C^{+}$, represented by a point of the moduli space $\bar{M}_{g^{+}, m}$ of pointed stable curves, with

$$
C=C^{+} \cup C_{0}^{-} .
$$

Note that while there is an ordering of the marked points of $C_{0}^{-}$, which is induced by the embedding of $C_{0}^{-}$into $C_{0}$, there is no such ordering of the marked points of $C^{+}$. The process of glueing with $C_{0}^{-}$determines the labels of the marked points of $C^{+}$only up to certain permutations, which are induced by automorphisms of $C_{0}^{-}$. Thus, while the curve $C^{+}$is not given as an $m$-pointed stable curve, it is uniquely determined as an $m / \Gamma$-pointed stable curve as introduced in [Z2], for a suitable group $\Gamma$. We will discuss this in detail in Section 3.

In this way we can identify the subscheme $D$ with a known moduli space $M$, see Lemma 3.4. Analogously, for the induced stratification of the moduli stack $\overline{\mathcal{M}}_{g}$ of Deligne-Mumford stable curves, there is a relationship between the connected components $\mathcal{D}$ of the one-dimensional stratum of stable curves with $3 g-4$ nodes, and corresponding moduli stacks $\mathcal{M}$. This relationship is our main topic, and the content of Section 6 .

As we will see, the stacks $\mathcal{D}$ and $\mathcal{M}$ are in general not isomorphic to each other, even though their associated moduli spaces are. The reason for this is that the group of automorphisms of a curve $C$ is usually larger than that of a subcurve $C^{+}$. This difference does not affect the moduli 
spaces, and therefore does not affect $D$ and $M$, but it has an impact on the associated moduli stacks. In fact, keeping track of automorphisms is what stacks are for. To recover an isomorphism, one essentially needs to enrich the stack $\mathcal{M}$ in order to take care of such automorphisms, which act trivially on the curves $C^{\prime}$ represented by points of $M$, but which act nontrivially on its complement in $C^{\prime} \cup C_{0}^{+}$.

For a general point of $D$ representing a curve $C$, its group of automorphisms $\operatorname{Aut}(C)$ splits in a natural way, see Section 3 below. This allows us to modify the moduli stack $\mathcal{M}$ by taking the quotient with respect to one of the factors of $\operatorname{Aut}(C)$. Our main result is Theorem 6.8, stating that a dense open substack $\mathcal{D}^{\times}$of $\mathcal{D}$ is isomorphic to a quotient of a dense open substack $\mathcal{M}^{\times}$of $\mathcal{M}$. In particular, the stack $\mathcal{D}$ can generically be constructed very explicitly as a quotient stack, and incidentally as a quotient of another more accessible moduli stack. We obtain a surjective morphism

$$
\mathcal{M}^{\times} \longrightarrow \mathcal{D}^{\times}
$$

and show that is unramified and finite of degree equal to the order of a certain group of automorphisms.

If the group $\operatorname{Aut}\left(C_{0}\right)$ splits, too, then with certain additional technical effort it is possible to construct an analogous isomorphism, which extends over the boundary point representing the curve $C_{0}^{+}$, i.e. above the point in the closure of $D$ which represents $C_{0}$. This is done in much detail in [Z1].

For a special curve $C$, the group of automorphisms $\operatorname{Aut}(C)$ may not split. For this reason it is in general not possible to extend the above morphism to all of $\mathcal{M}$. As explained in [Z1], it is in particular impossible to generalize Theorem 6.8 to a global quotient description of $\mathcal{D}$, if there are points in $D$ representing curves with non-splitting automorphism groups.

Our strategy to study the stacks $\mathcal{D}$ and $\mathcal{M}$ is to describe both as quotient stacks. We make use of the results of [Z2], where we generalized the construction of $\overline{\mathcal{M}}_{g}$ by Edidin [E] to moduli stacks of permutation classes of pointed stable curves. Using this quotient description, the relation between the stacks $\mathcal{M}$ and $\mathcal{D}$ is then given by a morphism between subschemes of suitable Hilbert schemes. It is straightforward to define a morphism $M \rightarrow D$ on the level of moduli spaces, using a process which Knudsen $[\mathrm{K}]$ called clutching. However, its lifting to the level of Hilbert schemes requires some extra work, which is done in the Sections 4 and 5. In particular, at this stage the automorphism groups of stable curves play an important part. 
To illustrate our results, we conclude this paper with a complete analysis of the one-dimensional stratum in the boundary of the moduli stack $\overline{\mathcal{M}}_{g}$ in the case of genus $g=3$. We compare our description to the classification Faber $[\mathrm{F}]$ gave in the case of the moduli scheme $\bar{M}_{3}$.

\section{$\S 2$. The moduli problem}

2.1. The curves we are interested in are Deligne-Mumford stable curves of some genus $g \geq 3$ in the sense of Knudsen $[K]$. He also introduced the more general notion of prestable curves. Here we will only be concerned with such prestable curves $f: C \rightarrow \operatorname{Spec}(k)$, where all connected components of $C$ are pointed stable curves themselves.

Notation 2.2. Throughout let one stable curve $f_{0}: C_{0} \rightarrow \operatorname{Spec}(k)$ of genus $g \geq 3$ be fixed, which attains the maximal number $3 g-3$ of nodes. We also choose an enumeration $P_{1}, \ldots, P_{3 g-3}$ of the nodes.

Let $C_{0}^{+}$denote the subscheme of the fixed curve $C_{0}$ which is the reduced union of all irreducible components of $C_{0}$ containing the node $P_{1}$. On $C_{0}^{+}$ there are marked points $Q_{1}, \ldots, Q_{m}$, where the $Q_{i} \in\left\{P_{2}, \ldots, P_{3 g-3}\right\}$ are all those points which are not singularities on $C_{0}^{+}$. Let $C_{0}^{-}$denote the closure of the complement of $C_{0}^{+}$in $C_{0}$, together with the same marked points $Q_{1}, \ldots, Q_{m}$. The set of all marked points shall be denoted by $\mathcal{Q}:=$ $\left\{Q_{1}, \ldots, Q_{m}\right\}$.

Note that if we allowed $g=2$, then there would exist nodes $P_{1}$, for which $C_{0}^{+}=C_{0}$ held, and thus $m=0$. To avoid this peculiarity, and to simplify our presentation, we exclude this otherwise analogous case.

2.3. Note that the chosen enumeration of the nodes of $C_{0}$ distinguishes an ordering of the marked points of the subcurves. Thus the subcurve $f_{0}^{+}: C_{0}^{+} \rightarrow \operatorname{Spec}(k)$ is in a natural way an $m$-pointed stable curve of some genus $g^{+}$. The subcurves $C_{0}^{+}$and $C_{0}^{-}$meet transversally, and exactly in the points $Q_{1}, \ldots, Q_{m}$. The curve $C_{0}^{-}$may have more than one connected component, so it is an $m$-pointed prestable curve of some genus $g^{-}$. There is an equality $g^{+}+g^{-}+m-1=g$. The connected components of $C_{0}^{-}$ are again pointed stable curves, each with the maximal number of nodes possible.

Remark 2.4. Only very few types of $m$-pointed stable curves $C_{0}^{+}$occur in this way, and here is a complete list. Note that each irreducible component of $C_{0}$ is a rational curve with at most three nodes of $C_{0}$ lying on it, so we must have $m \leq 4$. 
For $m=4$, there exist exactly three types of non-isomorphic nodal 4pointed stable curves of genus $g^{+}=0$, each consisting of two rational curves meeting each other transversally in one point.
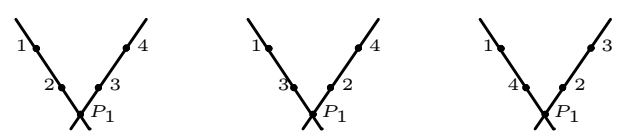

The curves pictured above are represented by the three points which lie in the boundary of the moduli space $\bar{M}_{0,4}$. If the condition on the existence of a node at $P_{1}$ is dropped, we obtain the full moduli space $\bar{M}_{0,4}$.

If $m=2$, then there exist two types of non-isomorphic nodal 2-pointed stable curves of genus $g^{+}=1$, both with two nodes and two rational irreducible components, as pictured below.
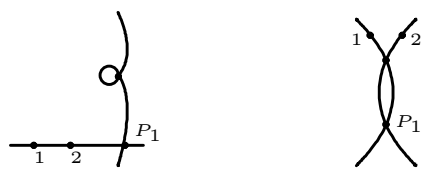

Both of them are represented by isolated points in the two-dimensional moduli space $\bar{M}_{1,2}$. The locus of curves in $\bar{M}_{1,2}$, which preserve the node which is not $P_{1}$, is a one-dimensional subscheme $\Delta_{0}$. In fact, $\Delta_{0}$ is given by that connected component of the boundary stratum $M_{1,2}^{(1)}$ of $\bar{M}_{1,2}$, which represents irreducible singular stable curves.

The only instance with $m=1$ is a curve of arithmetic genus $g^{+}=1$ with one node at $P_{1}$, i.e. the one point in the boundary of $\bar{M}_{1,1}$.

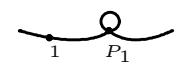

If the condition on the existence of a node at $P_{1}$ is dropped, we obtain the full moduli space $\bar{M}_{1,1}$.

Notation 2.5. For the integers $m=1,2$ or 4 which occur as numbers of marked points of $C_{0}^{-}$, we define

$$
M_{g^{+}, m}^{\prime}:= \begin{cases}M_{1,1} & \text { for } m=1 \\ \Delta_{0} & \text { for } m=2 \\ M_{0,4} & \text { for } m=4\end{cases}
$$


Here, as usual $M_{g^{+}, m}$ denotes the open subscheme of $\bar{M}_{g^{+}, m}$, parameterizing nonsingular stable curves. By $\Delta_{0} \subset \bar{M}_{1,2}$ we denote the reduced subscheme given by the locus of irreducible singular stable curves with exactly one node. Note that in each case the closure of $M_{g^{+}, m}^{\prime}$ is isomorphic to $\mathbb{P}^{1}$.

The pair $\left(C_{0}, P_{1}\right)$, which we fixed above, induces naturally a morphism

$$
\vartheta: M_{g^{+}, m}^{\prime} \longrightarrow \bar{M}_{g}
$$

as follows. For a point $[C] \in M_{g^{+}, m}^{\prime}$, represented by an $m$-pointed stable curve $f: C \rightarrow \operatorname{Spec}(k)$, its image $\vartheta([C])$ in $\bar{M}_{g}$ is defined as the point representing the stable curve of genus $g$ obtained by glueing $C$ and $C_{0}^{-}$in their respective marked points.

Since the scheme $M_{g^{+}, m}^{\prime}$ is connected, this morphism surjects onto one connected component of the boundary stratum $M_{g}^{(3 g-4)}$. By construction, the point representing $C_{0}$ is contained in the closure of the image of $\vartheta$.

Definition 2.6. Let a stable curve $f_{0}: C_{0} \rightarrow \operatorname{Spec}(k)$ with $3 g-3$ nodes and a node $P_{1}$ of $C_{0}$ be given. We denote by

$$
D\left(C_{0} ; P_{1}\right) \subset \bar{M}_{g}
$$

the connected component of the boundary stratum $M_{g}^{(3 g-4)}$, which is the reduced image of the above morphism $\vartheta$.

Decomposing a stable curve $f: C \rightarrow \operatorname{Spec}(k)$ by separating it at such nodes, where two irreducible components meet, is fairly straightforward. When we are dealing with families of curves, i.e. stable curves $f: C \rightarrow S$ over a general base scheme $S$, the situation becomes more subtle. To be able to "cut" along the nodes of $C$, the nodes of the fibres must be given by sections $\sigma: S \rightarrow C$, and in general this is not the case.

Lemma 2.7. Let $f: C \rightarrow S$ be a stable curve of genus $g$, with reduced and irreducible base $S$. Suppose that the number of nodes of the fibre over a general closed point is $k$, for some $1 \leq k \leq 3 g-3$. Then there exists an étale covering $u: S^{\prime} \rightarrow S$, where $S^{\prime}$ is connected, and such that the pullback $f^{\prime}: C^{\prime} \rightarrow S^{\prime}$ of $f: C \rightarrow S$ to $S^{\prime}$ admits $k$ disjoint sections of nodes.

Proof. Note that the assumption on a general fibre implies that for an arbitrary closed point $s \in S$, the fibre $C_{s}$ has at least $k$ nodes. Consider the 
subscheme $N \subset C$, which is defined as the reduced locus of all points of $C$ which are nodes of fibres of $f: C \rightarrow S$. Formally, it can be constructed as the locus of those points, where the sheaf $\Omega_{C / S}^{1}$ is not free. Let $p \in C$ be a point which is contained in some fibre $C_{s}$ over a point $s \in S$. Because of the flatness of $f: C \rightarrow S$, the sheaf $\Omega_{C / S}^{1}$ is free in this point if and only if the sheaf $\Omega_{C_{s}}^{1}$ is free in $p$. Therefore the points of $N$ are precisely the nodes of the fibres. Define $R$ as the reduced union of all those irreducible components of $N$, which surject onto $S$. Note that a node $n \in C_{s}$ is contained in $R$ if and only if there exists an open neighbourhood $U$ of $s$, such that the restriction $f: f^{-1}(U) \rightarrow U$ is a deformation of $C_{s}$ preserving the node $n$.

We claim that the restricted morphism $f \mid R: R \rightarrow S$ is an étale covering of $S$ of degree $k$. To see this, it suffices to show that for any point $s \in S$ its fibre consists of exactly $k$ simple points. Let us consider a general point $s \in S$ first. The $k$ nodes of the fibre $C_{s}$ persist in all neighbouring fibres, since by the assumption of the lemma the number of nodes is constant for all general fibres. Therefore all nodes of $C_{s}$ are contained in $R$, and the fibre of $f \mid R$ over $s$ consists exactly of those $k$ nodes. It remains to consider an arbitrary point $s \in S$. All nodes $n \in C_{s}$, which are contained in $R$, persist in neighbouring fibres. Thus the number of nodes of $C_{s}$ lying in $R$ may not exceed the number of nodes of a general fibre, which is $k$. Conversely, since there is no deformation, which splits one ordinary double point into two, the fibre of $f \mid R$ over $s$ must consist of at least as many points as a general fibre. This shows that for any point $s \in S$ the fibre of $f \mid R: R \rightarrow S$ consists of exactly $k$ simple points, as claimed.

By assumption, the base $S$ is connected. Therefore for any connected component $S_{1}$ of $R$ the restriction $g:=f \mid S_{1}: S_{1} \rightarrow S$ is an étale covering, too. We now define $f_{1}: C_{1} \rightarrow S_{1}$ as the pullback of $f: C \rightarrow S$ to $S_{1}$. In particular, the stable curve $f_{1}: C_{1} \rightarrow S_{1}$ admits a tautological section $\sigma_{1}: S_{1} \rightarrow C_{1}=S_{1} \times{ }_{S} C$, induced by the inclusion $S_{1} \subset C$, such that for all closed points $s \in S_{1}$, the point $\sigma_{1}(s)$ is a node of the fibre $C_{1, s}$.

Note that $\sigma\left(S_{1}\right)$ is a connected component of the pullback $g^{*} R \subset C_{1}$, since the restricted morphism $g^{*} R \rightarrow S_{1}$ is étale. Repeating the above construction with the complement $R_{1}:=g^{*} R \backslash \sigma\left(S_{1}\right)$ as a subscheme of $C_{1}$, we obtain an étale morphism $R_{1} \rightarrow S_{1}$ which is of degree $k-1$. Proceeding inductively, we define a sequence of étale coverings $S_{i+1} \rightarrow S_{i}$, for $i=$ $1, \ldots, k-1$, such that the combined pullback $f^{\prime}: C^{\prime} \rightarrow S^{\prime}$ of $f: C \rightarrow S$ to $S^{\prime}:=S_{k+1}$ admits $k$ sections of nodes. 


\section{§3. Automorphisms of decomposing stable curves}

The automorphisms of stable curves will play an important part in our considerations below. We need to relate the group of automorphisms of a stable curve to the automorphism groups of its subcurves. In particular we need to understand the group $\operatorname{Aut}\left(C_{0}^{-}\right)$.

Notation 3.1. Let $\mathcal{Q}:=\left\{Q_{1}, \ldots, Q_{m}\right\}$ denote the set of marked points of an $m$-pointed curve $f: C \rightarrow \operatorname{Spec}(k)$, like for example $C_{0}^{-}$or $C_{0}^{+}$. We define $\operatorname{Aut}(C ; \mathcal{Q})$ as the group of all those automorphisms of $C$ as a scheme over $\operatorname{Spec}(k)$, which map the set $\mathcal{Q}$ to itself. As usual, $\operatorname{Aut}(C)$ denotes the automorphism group of $C$ as an $m$-pointed curve.

Let $\Sigma_{m}$ denote the permutation group acting on the labels of the $m$ marked points of $C$ contained in $\mathcal{Q}$. For any $\phi \in \operatorname{Aut}(C ; \mathcal{Q})$ there is a unique permutation $\gamma \in \Sigma_{m}$ such that $\phi\left(Q_{i}\right)=Q_{\gamma(i)}$ holds for all $i=1, \ldots, m$. Thus there is a natural homomorphism of groups $\operatorname{Aut}(C ; \mathcal{Q}) \rightarrow \Sigma_{m}$. We define $\Gamma(C ; \mathcal{Q})$ as the image of this homomorphism. In particular there is an exact sequence

$$
\mathrm{id} \longrightarrow \operatorname{Aut}(C) \longrightarrow \operatorname{Aut}(C ; \mathcal{Q}) \longrightarrow \Gamma(C ; \mathcal{Q}) \longrightarrow \mathrm{id}
$$

Let $\Gamma \subset \Sigma_{m}$ be a subgroup. By definition, an $m / \Gamma$-pointed stable curve over $\operatorname{Spec}(k)$ is an equivalence class of $m$-pointed stable curves $f: C \rightarrow$ $\operatorname{Spec}(k)$ with respect to the action of $\Gamma$ on the labels of the marked points, as introduced in [Z2]. It is also called a permutation class of stable curves. The group of automorphisms of the $m / \Gamma$-pointed stable curve represented by $C$ is denoted by $\operatorname{Aut}_{\Gamma}(C ; \mathcal{Q})$. It is the subgroup of those elements $\phi \in \operatorname{Aut}(C ; \mathcal{Q})$, for which there exists a permutation $\gamma \in \Gamma$ such that $\phi\left(Q_{i}\right)=Q_{\gamma(i)}$ holds for all $i=1, \ldots, m$.

Lemma 3.2. Let $f^{+}: C^{+} \rightarrow \operatorname{Spec}(k)$ be a stable curve of genus $g^{+}$ with $m$ marked points, which is represented by a point $\left[C^{+}\right] \in M_{g^{+}, m}^{\prime}$. Let $f: C \rightarrow \operatorname{Spec}(k)$ be the stable curve of genus $g$, which is obtained by glueing $C^{+}$with $C_{0}^{-}$in the $m$ marked points. Then there is an exact sequence of groups

$$
\mathrm{id} \longrightarrow \operatorname{Aut}\left(C_{0}^{-}\right) \longrightarrow \operatorname{Aut}(C) \longrightarrow \operatorname{Aut}_{\Gamma}\left(C^{+} ; \mathcal{Q}\right) \longrightarrow \mathrm{id}
$$

where $\Gamma=\Gamma\left(C_{0}^{-} ; \mathcal{Q}\right)$. 
Proof. By definition, any automorphism in $\operatorname{Aut}\left(C_{0}^{-}\right)$fixes each one of the marked points of $C_{0}^{-}$, and these marked points are the glueing points of $C_{0}^{-}$with $C^{+}$. By trivial extension, the group $\operatorname{Aut}\left(C_{0}^{-}\right)$becomes a subgroup of $\operatorname{Aut}(C)$. Conversely, since $\left[C^{+}\right] \in M_{g^{+}, m}^{\prime}$, the curve $C^{+}$is the unique connected subcurve of $C$ of genus $g^{+}$, which has not the maximal number of nodes as an $m$-pointed stable curve. Hence any automorphism of $C$ restricts to an automorphism on $C_{0}^{-}$and to an automorphism on $C^{+}$, which both are not necessarily fixing the marked points. However, in this way only such permutations of marked points occur on $C^{+}$, which are also effected by automorphisms on $C_{0}^{-}$. Thus by definition, an automorphism of $C$ restricted to $C^{+}$is an automorphism of $C^{+}$considered as an $m / \Gamma$-pointed stable curve, i.e. an element of $\operatorname{Aut}_{\Gamma}\left(C^{+} ; \mathcal{Q}\right)$.

All automorphisms in $\operatorname{Aut}_{\Gamma}\left(C^{+} ; \mathcal{Q}\right)$ occur in this way. To see this, let $\varrho^{+} \in \operatorname{Aut}_{\Gamma}\left(C^{+} ; \mathcal{Q}\right)$ be an automorphism of $C^{+}$as an $m / \Gamma$-pointed stable curve. It induces a permutation $\gamma$ of the marked points, with $\gamma \in \Gamma$. By the definition of $\Gamma$, there exists an automorphism $\varrho^{-} \in \operatorname{Aut}\left(C_{0}^{-} ; \mathcal{Q}\right)$ inducing $\gamma$. Glueing the automorphisms $\varrho^{+}$and $\varrho^{-}$defines an automorphism $\varrho$ on $C$, which restricts to $\varrho^{+}$. Thus we have shown that there exists an exact sequence of groups, as claimed.

Remark 3.3. For a general point $\left[C^{+}\right] \in M_{g^{+}, m}^{\prime}$ as in Lemma 3.2, the exact sequence of the lemma splits in a natural way. This can be seen as follows. If $m=1$, then clearly $\operatorname{Aut}_{\Gamma}\left(C^{+} ; \mathcal{Q}\right)=\operatorname{Aut}\left(C^{+}\right)$. If $m=2$ or $m=4$, then an examination of the list of possibilities for curves $C^{+}$from Remark 2.4 shows that automorphisms of $C^{+}$, which permute the marked points, exist only if the marked points are in a very special position. Thus, if $\left[C^{+}\right] \in M_{g^{+}, m}^{\prime}$ is a general point, then again $\operatorname{Aut}_{\Gamma}\left(C^{+} ; \mathcal{Q}\right)=\operatorname{Aut}\left(C^{+}\right)$. Now a splitting homomorphism $\operatorname{Aut}_{\Gamma}\left(C^{+} ; \mathcal{Q}\right) \rightarrow \operatorname{Aut}(C)$ can be defined by trivial extension.

Lemma 3.4. Let $f: C \rightarrow \operatorname{Spec}(k)$ be a stable curve of genus $g \geq 3$ represented by a point $[C] \in D\left(C_{0} ; P_{1}\right)$. Let $\Gamma=\Gamma\left(C_{0}^{-} ; \mathcal{Q}\right)$. Then there exists a uniquely determined $m / \Gamma$-pointed stable curve $C^{+}$, with $m \in\{1,2,4\}$, such that $C$ is obtained from glueing a representative of $C^{+}$and the $m$-pointed prestable curve $C_{0}^{-}$in the corresponding marked points. In particular, there is an isomorphism of schemes

$$
D\left(C_{0} ; P_{1}\right) \cong M_{g^{+}, m}^{\prime} / \Gamma
$$


Let us remark that the closure of $D\left(C_{0} ; P_{1}\right)$ in $\bar{M}_{g}$ is always isomorphic to $\mathbb{P}^{1}$, see $[\mathrm{Z} 1]$.

Proof. By the definition of $D\left(C_{0} ; P_{1}\right)$, there exists a decomposition of the curve $C$ into an $m$-pointed stable subcurve $C^{+}$and a subcurve isomorphic to $C_{0}^{-}$. The labels of the marked points of $C^{+}$are determined only up to certain permutations, which are induced by automorphisms of $C_{0}^{-}$, i.e. $\mathrm{C}^{+}$is uniquely determined as an $m / \Gamma$-pointed stable curve.

Definition 3.5. We define the subscheme

$$
D^{\times}\left(C_{0} ; P_{1}\right) \subset D\left(C_{0} ; P_{1}\right)
$$

as the nonempty reduced open subscheme, which is the locus of points $[C]$ parameterizing those curves $f: C \rightarrow \operatorname{Spec}(k)$, whose groups of automorphisms split naturally as $\operatorname{Aut}(C)=\operatorname{Aut}_{\Gamma}\left(C^{+} ; \mathcal{Q}\right) \times \operatorname{Aut}\left(C_{0}^{-}\right)$, where $f^{+}: C^{+} \rightarrow \operatorname{Spec}(k)$ denotes the unique subcurve as in Lemma 3.4.

\section{§. The glueing morphism}

Our objective in this section is to construct a lifting of the natural morphism $\vartheta: M_{g^{+}, m}^{\prime} \rightarrow \bar{M}_{g}$ from paragraph 2.5 generically to a morphism between subschemes of Hilbert schemes.

Notation 4.1. Let $f: C \rightarrow S$ be an $m$-pointed stable curve of genus $g$ over some base scheme $S$, such that $2 g-2+m>0$. Let $S_{1}, \ldots, S_{m}$ denote the divisors of marked points of $C$, and let $\omega_{C / S}$ be the canonical sheaf. If $n \in \mathbb{N}$ is chosen large enough, then the sheaf $\left(\omega_{C / S}\left(S_{1}+\cdots+S_{m}\right)\right)^{\otimes n}$ is relatively very ample, and $f_{*}\left(\left(\omega_{C / S}\left(S_{1}+\cdots+S_{m}\right)\right)^{\otimes n}\right)$ is locally free of rank $(2 g-2+m) n-g+1$, compare $[\mathrm{K}]$.

Consider the Hilbert scheme $\operatorname{Hilb}_{\mathbb{P}^{N}}^{P_{g, n, m}}$ of curves $f: C \rightarrow \operatorname{Spec}(k)$ embedded in $\mathbb{P}^{N}$ with Hilbert polynomial $P_{g, n, m}(t):=(2 g-2+m) n t-g+1$, where $N:=(2 g-2+m) n-g$. We denote by

$$
\bar{H}_{g, n, m} \subset \operatorname{Hilb}_{\mathbb{P}^{N}}^{P_{g, n, m}} \times\left(\mathbb{P}^{N}\right)^{m}
$$

the quasi-projective reduced subscheme, which is the locus of $n$-canonically embedded $m$-pointed stable curves.

The standard construction of the moduli space $\bar{M}_{g, m}$ of $m$-pointed stable curves of genus $g$ is its construction as the GIT-quotient of $\bar{H}_{g, n, m}$ with 
respect to the natural $\operatorname{PGL}(N+1)$-action, compare for example $[\mathrm{DM}]$ or $[\mathrm{G}]$.

If $\Gamma \subset \Sigma_{m}$ is a subgroup of the group of permutations on the labels of the $m$ marked points, then the moduli space of $m / \Gamma$-pointed stable curves is the quotient $\bar{M}_{g, m / \Gamma}:=\bar{M}_{g, m} / \Gamma$, see [Z2]. Equivalently, it can be constructed as the GIT-quotient of $\bar{H}_{g, n, m / \Gamma}:=\bar{H}_{g, n, m} / \Gamma$ with respect to the natural $\operatorname{PGL}(N+1)$-action.

4.2. As before, let $f_{0}: C_{0} \rightarrow \operatorname{Spec}(k)$ denote the fixed stable curve of genus $g \geq 3$ with $3 g-3$ nodes, and let $P_{1}, \ldots, P_{3 g-3}$ be an enumeration of its nodes. Let $C_{0}^{-}$denote the unique $m$-pointed prestable curve of genus $g^{-}$, which is the reduced union of all of the irreducible components of $C_{0}$ which do not contain $P_{1}$. The closure of the complement of $C_{0}^{-}$in $C_{0}$ is denoted by $C_{0}^{+}$. It can be viewed as an $m$-pointed stable curve of some genus $g^{+}$.

Notation 4.3. Let $\Gamma:=\Gamma\left(C_{0}^{-} ; \mathcal{Q}\right)$. For $n \in \mathbb{N}$ sufficiently large let $\bar{H}_{g, n, 0}$ and $\bar{H}_{g^{+}, n, m / \Gamma}$ denote the subschemes of the Hilbert schemes as in 4.1, with the moduli spaces $\bar{M}_{g}$ and $\bar{M}_{g^{+}, m / \Gamma}$ as their respective GIT-quotients. Let $H_{g^{+}, n, m}^{\prime}$ denote the reduced preimage of $M_{g^{+}, m}^{\prime}$ in $\bar{H}_{g^{+}, n, m}$, and we define $H_{g^{+}, n, m / \Gamma}^{\prime \prime, m}:=H_{g^{+}, n, m}^{\prime} / \Gamma$, as well as $M_{g^{+}, m / \Gamma}^{\prime}:=M_{g^{+}, m}^{\prime} / \Gamma$.

4.4. Construction step 1. Consider the universal embedded $m$-pointed stable curve $u^{+}: \mathcal{C}_{g^{+}, n, m} \rightarrow \bar{H}_{g^{+}, n, m}$, which is the restriction of the universal curve on the full Hilbert scheme. We glue this along the $m$ given sections to the trivial $m$-pointed prestable curve $C_{0}^{-} \times \bar{H}_{g^{+}, n, m} \rightarrow \bar{H}_{g^{+}, n, m}$. This produces a stable curve $u_{0}: \mathcal{C}_{0} \rightarrow \bar{H}_{g^{+}, n, m}$ of genus $g$, compare [K]. Knudsen calls this procedure of glueing along a pair of disjoint sections "clutching".

Let $i: \mathcal{C}_{g^{+}, n, m} \hookrightarrow \mathcal{C}_{0}$ denote the inclusion morphism. Consider the restriction of the canonical invertible dualizing sheaf $\omega_{\mathcal{C}_{0} / \bar{H}_{g^{+}, n, m}}$ to a fibre $C_{h} \rightarrow \operatorname{Spec}(k)$ of $u_{0}: \mathcal{C}_{0} \rightarrow \bar{H}_{g^{+}, n, m}$, for some closed point $h \in \bar{H}_{g^{+}, n, m}$. Let $n: \hat{C}_{h} \rightarrow C_{h}$ be the normalization of $C_{h}$, and for a node $P_{i} \in C_{h}$ let $A_{i}$ and $B_{i}$ denote its preimages in $\hat{C}_{h}$. The restriction of $\omega_{\mathcal{C}_{0} / \bar{H}_{g^{+}, n, m}}$ to $C_{h}$ can be described as the sheaf of those 1 -forms on $\hat{C}_{h}$ which have at most simple poles in $A_{i}$ and $B_{i}$, and such that the sum of the residues in $A_{i}$ and $B_{i}$ is zero, compare for example $[\mathrm{K}]$.

By construction, the curve $u_{0}: \mathcal{C}_{0} \rightarrow \bar{H}_{g^{+}, n, m}$ comes with $m$ disjoint sections of nodes, which are given by the sections of glueing. Let $n_{0}: \hat{C}_{h}^{\prime} \rightarrow C_{h}$ 
denote the partial normalization of $C_{h}$, in the sense that it is the normalization map near those nodes resulting from the glueing, and an isomorphism elsewhere. In particular, $\hat{C}_{h}^{\prime}$ preserves exactly those nodes, which are not glueing nodes. Thus $\hat{C}_{h}^{\prime}$ decomposes into two disjoint subcurves, one of them isomorphic to $C_{0}^{-}$, the other one isomorphic to $C_{h}^{+}$, where $C_{h}^{+}$is the fibre of the universal curve $u^{+}: \mathcal{C}_{g^{+}, n, m} \rightarrow \bar{H}_{g^{+}, n, m}$ over the point $h$. This induces a morphism $\iota: C_{h}^{+} \rightarrow \hat{C}_{h}^{\prime}$, such that $i=n_{0} \circ \iota$, and an isomorphism $\iota^{*} n_{0}^{*} \omega_{C_{h} / k} \cong \omega_{C_{h}^{+} / k}\left(Q_{1}+\ldots+Q_{m}\right)$, where $Q_{1}, \ldots, Q_{m}$ denote the marked points on $C_{h}^{+}$. Thus there is a natural isomorphism of sheaves

$$
i^{*} \omega_{\mathcal{C}_{0} / \bar{H}_{g^{+}, n, m}} \cong \omega_{\mathcal{C}_{g^{+}, n, m} / \bar{H}_{g^{+}, n, m}}\left(\mathcal{S}_{1}+\cdots+\mathcal{S}_{m}\right) .
$$

There is a natural surjection of sheaves $\omega_{\mathcal{C}_{0} / \bar{H}_{g^{+}, n, m}} \rightarrow i_{*} i^{*} \omega_{\mathcal{C}_{0} / \bar{H}_{g^{+}, n, m}}$. After applying $n$-th tensor powers, and push-forward, we obtain a surjection

$$
\left(u_{0}\right)_{*}\left(\omega_{\mathcal{C}_{0} / \bar{H}_{g^{+}, n, m}}\right)^{\otimes n} \longrightarrow u_{*}^{+} i^{*}\left(\omega_{\mathcal{C}_{0} / \bar{H}_{g^{+}, n, m}}\right)^{\otimes n}
$$

of locally free sheaves, using the factorization $u^{+}=u_{0} \circ i$. Together with the above isomorphism, and applying Grothendieck's construction of the associated projective bundle, we obtain an inclusion of projective bundles

$$
\mathbb{P} u_{*}^{+}\left(\omega_{\mathcal{C}_{g^{+}, n, m} / \bar{H}_{g^{+}, n, m}}\left(\mathcal{S}_{1}+\cdots+\mathcal{S}_{m}\right)\right)^{\otimes n} \longleftrightarrow \mathbb{P}\left(u_{0}\right)_{*}\left(\omega_{\mathcal{C}_{0} / \bar{H}_{g^{+}, n, m}}\right)^{\otimes n} .
$$

Recall that $f_{0}: C_{0} \rightarrow \operatorname{Spec}(k)$ is the stable curve fixed throughout this section, and $f_{0}^{+}: C_{0}^{+} \rightarrow \operatorname{Spec}(k)$ is the subcurve defined in 2.2. We fix once and for all an embedding of $C_{0}$ into $\mathbb{P}^{N}$, which is equivalent to distinguishing an isomorphism $\mathbb{P}\left(f_{0}\right)_{*}\left(\omega_{C_{0} / k}\right)^{\otimes n} \cong \mathbb{P}^{N}$, and we fix an embedding of $C_{0}^{+}$into $\mathbb{P}^{N^{+}}$, with $N^{+}:=\left(2 g^{+}-2+m\right) n-g^{+}+1$, corresponding to an isomorphism $\mathbb{P}\left(f_{0}^{+}\right)_{*}\left(\omega_{C_{0}^{+} / k}\left(Q_{1}+\cdots+Q_{m}\right)\right)^{\otimes n} \cong \mathbb{P}^{N^{+}}$, in such a way that the diagram

$$
\begin{array}{cccccc}
C_{0}^{+} & \hookrightarrow & \mathbb{P}^{N^{+}} & \cong & \mathbb{P}\left(f_{0}^{+}\right)_{*}\left(\omega_{C_{0}^{+} / k}\left(Q_{1}+\cdots+Q_{m}\right)\right)^{\otimes n} \\
\downarrow & & \downarrow & & \downarrow \\
C_{0} & \hookrightarrow & \mathbb{P}^{N} & \cong & \mathbb{P}\left(f_{0}\right)_{*}\left(\omega_{C_{0} / k}\right)^{\otimes n}
\end{array}
$$

commutes. The universal curve $u^{+}: \mathcal{C}_{g^{+}, n, m} \rightarrow \bar{H}_{g^{+}, n, m}$ determines a trivialization

$$
\begin{array}{r}
\mathbb{P} u_{*}^{+}\left(\omega_{\mathcal{C}_{g^{+}, n, m} / \bar{H}_{g^{+}, n, m}}\left(\mathcal{S}_{1}+\cdots+\mathcal{S}_{m}\right)\right)^{\otimes n} \cong \mathbb{P}^{N^{+}} \times \bar{H}_{g^{+}, n, m} \\
\cong \mathbb{P}\left(f_{0}^{+}\right)_{*}\left(\omega_{C_{0}^{+} / k}\left(Q_{1}+\cdots+Q_{m}\right)\right)^{\otimes n} \times \bar{H}_{g^{+}, n, m} .
\end{array}
$$


The tautological section of this projective bundle shall be denoted by

$$
\tau^{+}: \bar{H}_{g^{+}, n, m} \longrightarrow \mathbb{P} u_{*}^{+}\left(\omega_{\mathcal{C}_{g^{+}, n, m}} / \bar{H}_{g^{+}, n, m}\left(\mathcal{S}_{1}+\cdots+\mathcal{S}_{m}\right)\right)^{\otimes n} .
$$

If $\left[C^{+}\right] \in \bar{H}_{g^{+}, n, m}$ is a point representing an embedded curve $C^{+}$, then by definition of $\tau^{+}$, the point $\tau^{+}\left(\left[C^{+}\right]\right)$is fixed under the action of the group $\operatorname{Aut}\left(C^{+}\right) \cong \operatorname{Stab}_{\mathrm{PGL}\left(N^{+}+1\right)}\left(\left[C^{+}\right]\right)$on the fibre of the projective bundle over the point $\left[C^{+}\right]$.

Remark 4.5. Via diagram $(\diamond)$ of the above construction, we may consider $\operatorname{PGL}\left(N^{+}+1\right)$ as a subgroup of $\operatorname{PGL}(N+1)$. The group of automorphisms of $C_{0}$ can be identified with a subgroup of $\operatorname{PGL}(N+1)$ via $\operatorname{Aut}\left(C_{0}\right) \cong \operatorname{Stab}_{\mathrm{PGL}(N+1)}\left(\left[C_{0}\right]\right)$, and the diagram

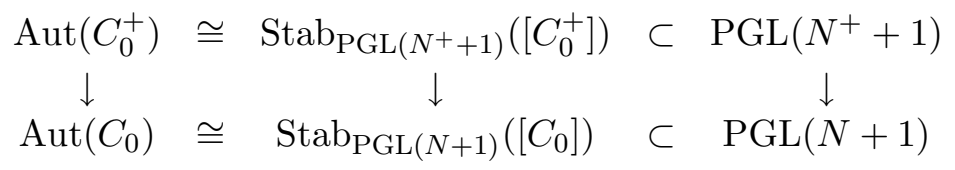

commutes. Furthermore, for any embedded curve $C^{+}$, which is represented by a point $\left[C^{+}\right] \in \bar{H}_{g^{+}, n, m}$, there is a natural embedding of $\operatorname{Aut}\left(C^{+}\right)$first into the group $\operatorname{PGL}\left(N^{+}+1\right)$, and from there into $\operatorname{PGL}(N+1)$.

4.6. Construction step 2. As in 4.4, there is also a natural surjection of the sheaves $j^{*} \omega_{\mathcal{C}_{0} / H_{g^{+}, n, m}} \rightarrow \omega_{C_{0}^{-} \times \bar{H}_{g^{+}, n, m} / \bar{H}_{g^{+}, n, m}}\left(\mathcal{S}_{1}+\cdots+\mathcal{S}_{m}\right)$, where $j: C_{0}^{-} \times \bar{H}_{g^{+}, n, m} \rightarrow \mathcal{C}_{0}$ denotes the embedding as a subcurve over $\bar{H}_{g^{+}, n, m}$ as constructed above. Clearly there is a trivialization

$$
\mathbb{P}^{N^{-}} \times \bar{H}_{g^{+}, n, m} \cong \mathbb{P}\left(\left(\operatorname{pr}_{2}\right)_{*}\left(\omega_{C_{0}^{-} \times \bar{H}_{g^{+}, n, m}} / \bar{H}_{g^{+}, n, m}\left(\mathcal{S}_{1}+\cdots+\mathcal{S}_{m}\right)\right)^{\otimes n}\right)
$$

as a projective bundle over $\bar{H}_{g^{+}, n, m}$, where $N^{-}=\left(2 g^{-}-2+m\right) n-g^{-}+1$, provided $n$ has been chosen sufficiently large. We may assume that the corresponding embedding of $C_{0}^{-}$into $\mathbb{P}^{N^{-}}$is generic in the sense that the $m$ marked points on $C_{0}^{-}$are contained in no subspace of dimension $m-2$. The tautological section shall be denoted by $\tau^{-}$.

The above projective bundle is again in a natural way a subbundle of the projective bundle $\mathbb{P}\left(u_{0}\right)_{*}\left(\omega_{\mathcal{C}_{0} / \bar{H}_{g^{+}, n, m}}\right)^{\otimes n}$. 
4.7. Construction step 3. Combining the constructions of 4.4 and 4.6, we obtain two subbundles of $\mathbb{P}\left(u_{0}\right)_{*}\left(\omega_{\mathcal{C}_{0} / \bar{H}_{g^{+}, n, m}}\right)^{\otimes n}$, together with a surjective morphism $\pi$ :

$$
\begin{aligned}
& \mathbb{P} u_{*}^{+}\left(\omega_{\mathcal{C}_{g^{+}, n, m} / \bar{H}_{g^{+}, n, m}}\left(\sum_{i=1}^{m} \mathcal{S}_{i}\right)\right)^{\otimes n} \\
& \mathbb{P}\left(\operatorname{pr}_{2}\right)_{*}\left(\omega_{C_{0}^{-} \times \bar{H}_{g^{+}, n, m} / \bar{H}_{g^{+}, n, m}}\left(\sum_{i=1}^{m} \mathcal{S}_{i}\right)\right)^{\otimes n} \stackrel{\pi}{\longrightarrow} \mathbb{P}\left(u_{0}\right)_{*}\left(\omega_{\mathcal{C}_{0} / \bar{H}_{g^{+}, n, m}}\right)^{\otimes n}
\end{aligned}
$$

of projective bundles over $\bar{H}_{g^{+}, n, m}$. Note that the intersection of the two subbundles can be described as the projectivization of the cokernel of an injective homomorphism

$$
\begin{gathered}
u_{*}^{+}\left(\omega_{\mathcal{C}_{g^{+}, n, m} / \bar{H}_{g^{+}, n, m}}\left(\mathcal{S}_{1}+\cdots+\mathcal{S}_{m}\right)\right)^{\otimes n}\left(\omega_{\mathcal{C}_{0} / \bar{H}_{g^{+}, n, m}}\right)^{\otimes n} \rightarrow \underbrace{\oplus}_{\left(\operatorname{pr}_{2}\right)_{*}\left(\omega_{C_{0}^{-} \times \bar{H}_{g^{+}, n, m} / \bar{H}_{g^{+}, n, m}}\left(\mathcal{S}_{1}+\cdots+\mathcal{S}_{m}\right)\right)^{\otimes n}}
\end{gathered}
$$

of locally free sheaves over the base $\bar{H}_{g^{+}, n, m}$.

Thus, for a fibre of $u_{0}: \mathcal{C}_{0} \rightarrow \bar{H}_{g^{+}, n, m}$, the subcurve $C_{0}^{-}$is embedded into a subspace $\mathbb{P}^{N^{-}} \subset \mathbb{P}^{N}$, while the closure of its complement is embedded into a subspace $\mathbb{P}^{N^{+}} \subset \mathbb{P}^{N}$, and the intersection of $\mathbb{P}^{N^{-}}$and $\mathbb{P}^{N^{+}}$is a subspace of dimension $m-1$. This is just the subspace spanned by the $m$ marked points of $C_{0}^{-}$embedded into $\mathbb{P}^{N}$.

By construction, for the two sections of the subbundles holds $\pi \circ \tau^{+}=$ $\pi \circ \tau^{-}$, considered as a section from $\bar{H}_{g^{+}, n, m}$ into the bundle $\mathbb{P}\left(u_{0}\right)_{*}\left(\omega_{\mathcal{C}_{0} / \bar{H}_{g^{+}, n, m}}\right)^{\otimes n}$. In 4.10 we will use this section to construct a trivialization of the ambient $\operatorname{PGL}(N+1)$-bundle, extending the trivializations of the subbundles.

Remark 4.8. In exactly the same way as in Remark 4.5, the construction of paragraph 4.6 induces an embedding of $\operatorname{PGL}\left(N^{-}+1\right)$ into $\operatorname{PGL}(N+$ 1). In particular, the group $\operatorname{Aut}\left(C_{0}^{-} ; \mathcal{Q}\right)$ can be considered as a subgroup of $\operatorname{PGL}(N+1)$, too.

Notation 4.9. We define the nonempty reduced subscheme

$$
H_{g^{+}, n, m}^{\times} \subset \bar{H}_{g^{+}, n, m}
$$

of the Hilbert scheme as the locus of points $\left[C^{+}\right] \in H_{g^{+}, n, m}^{\prime}$ parameterizing curves $f^{+}: C^{+} \rightarrow \operatorname{Spec}(k)$ with the following property: if $f: C \rightarrow \operatorname{Spec}(k)$ 
denotes the stable curve of genus $g$, which is obtained by glueing $C^{+}$with $C_{0}^{-}$ in the $m$ marked points, then its group of automorphisms splits naturally as $\operatorname{Aut}(C)=\operatorname{Aut}_{\Gamma}\left(C^{+} ; \mathcal{Q}\right) \times \operatorname{Aut}\left(C_{0}^{-}\right)$. It follows from Remark 3.3 that $H_{g^{+}, n, m}^{\times}$is a dense subscheme of $H_{g^{+}, n, m}^{\prime}$.

Let $M_{g^{+}, m}^{\times}$denote the GIT-quotient of $H_{g^{+}, n, m}^{\times}$with respect to the natural $\operatorname{PGL}\left(N^{+}+1\right)$-action. For $\Gamma=\Gamma\left(C_{0}^{-} ; \mathcal{Q}\right)$ we define $H_{g^{+}, n, m / \Gamma}^{\times}:=$ $H_{g^{+}, n, m}^{\times} / \Gamma$ and $M_{g^{+}, m / \Gamma}^{\times}:=M_{g^{+}, m}^{\times} / \Gamma$.

4.10. Construction step 4. Let $\pi$ denote the morphism of projective bundles over $\bar{H}_{g^{+}, n, m}$ as in 4.7. The restriction of $\pi$ to $H_{g^{+}, n, m}^{\times}$, composed with the tautological sections $\tau^{+}$and $\tau^{-}$of the subbundles, respectively, defines in both cases the same section

$$
\tau: H_{g^{+}, n, m}^{\times} \longrightarrow \mathbb{P}\left(u_{0}\right)_{*}\left(\omega_{\mathcal{C}_{0} / \bar{H}_{g^{+}, n, m}}\right)^{\otimes n} \mid H_{g^{+}, n, m}^{\times},
$$

compare 4.7. By 4.4 and 4.6, there exist trivializations

$$
\vartheta^{+}: \mathbb{P} u_{*}^{+}\left(\omega_{\mathcal{C}_{g^{+}, n, m} / \bar{H}_{g^{+}, n, m}}\left(\sum_{i=1}^{m} \mathcal{S}_{i}\right)\right)^{\otimes n} \mid H_{g^{+}, n, m}^{\times} \longrightarrow \mathbb{P}^{N^{+}} \times H_{g^{+}, n, m}^{\times}
$$

and

$\vartheta^{-}: \mathbb{P}\left(\operatorname{pr}_{2}\right)_{*}\left(\omega_{C_{0}^{-} \times \bar{H}_{g^{+}, n, m} / \bar{H}_{g^{+}, n, m}}\left(\sum_{i=1}^{m} \mathcal{S}_{i}\right)\right)^{\otimes n} \mid H_{g^{+}, n, m}^{\times} \longrightarrow \mathbb{P}^{N^{+}} \times H_{g^{+}, n, m}^{\times}$

as $\operatorname{PGL}\left(N^{+}+1\right)$-bundles and $\operatorname{PGL}\left(N^{-}+1\right)$-bundles, respectively. We want to extend both of these to a common trivialization of the ambient $\operatorname{PGL}(N+$ 1)-bundle $\mathbb{P}\left(u_{0}\right)_{*}\left(\omega_{\mathcal{C}_{0} / \bar{H}_{g^{+}, n, m}}\right)^{\otimes n} \mid H_{g^{+}, n, m}^{\times}$. If $e$ is a point in the fibre of $\mathbb{P}\left(u_{0}\right)_{*}\left(\omega_{\mathcal{C}_{0} / \bar{H}_{g^{+}, n, m}}\right)^{\otimes n} \mid H_{g^{+}, n, m}^{\times}$over a point $\left[C^{+}\right] \in H_{g^{+}, n, m}^{\times}$, representing an embedded $m$-pointed stable curve $C^{+}$, then $e$ can be written as $e=$ $\gamma \cdot \tau\left(\left[C^{+}\right]\right)$for some $\gamma \in \operatorname{PGL}(N+1)$. We now define

$$
\vartheta: \mathbb{P}\left(u_{0}\right)_{*}\left(\omega_{\mathcal{C}_{0} / \bar{H}_{g^{+}, n, m}}\right)^{\otimes n} \mid H_{g^{+}, n, m}^{\times} \longrightarrow \mathbb{P}^{N} \times H_{g^{+}, n, m}^{\times}
$$

by $\vartheta(e):=\gamma \cdot \vartheta^{+}\left(\tau^{+}\left(\left[C^{+}\right]\right)\right)$, using the fact that the point $\tau\left(\left[C^{+}\right]\right)=\pi \circ$ $\tau^{+}\left(\left[C^{+}\right]\right)$is contained in the subbundle $\mathbb{P} u_{*}^{+}\left(\omega_{\mathcal{C}_{g^{+}, n, m}} / \bar{H}_{g^{+}, n, m}\left(\sum_{i=1}^{m} \mathcal{S}_{i}\right)\right)^{\otimes n}$, and the fixed embedding of the subspace $\mathbb{P}^{N^{+}} \subset \mathbb{P}^{N}$ from 4.4. Note that in general $\gamma$ is not uniquely determined by the point $e$. To show that the above 
map is indeed well-defined, it suffices to show that all elements of $\operatorname{PGL}(N+$ $1)$, which stabilize the point $\tau\left(\left[C^{+}\right]\right)$also stabilize the point $\vartheta^{+}\left(\tau^{+}\left(\left[C^{+}\right]\right)\right)$. By construction, the elements stabilizing the point $\tau\left(\left[C^{+}\right]\right)$in the fibre are exactly the elements of the group $\operatorname{Aut}(C) \cong \operatorname{Stab}_{\mathrm{PGL}(N+1)}([C])$, where $C$ is the embedded curve in $\mathbb{P}^{N}$ obtained by glueing $C^{+}$and $C_{0}^{-}$in their marked points. Recall that $\tau=\pi \circ \tau^{+}=\pi \circ \tau^{-}$, and thus $\vartheta^{+}\left(\tau^{+}\left(\left[C^{+}\right]\right)\right)=$ $\vartheta^{-}\left(\tau^{-}\left(\left[C^{+}\right]\right)\right) \in \mathbb{P}^{N}$, with respect to the fixed embeddings of 4.4 and 4.6 .

By the definition of the subscheme $H_{g^{+}, n, m}^{\times}$we have a splitting of $\operatorname{Aut}(C)$ as a product $\operatorname{Aut}_{\Gamma}\left(C^{+} ; \mathcal{Q}\right) \times \operatorname{Aut}\left(C_{0}^{-}\right)$. Because $\tau^{+}$and $\tau^{-}$are the tautological sections of the subbundles, the points $\vartheta^{+}\left(\tau^{+}\left(\left[C^{+}\right]\right)\right)$and $\vartheta^{-}\left(\tau^{-}\left(\left[C^{+}\right]\right)\right)$ are fixed by $\operatorname{Aut}\left(C^{+}\right)$and $\operatorname{Aut}\left(C_{0}^{-}\right)$, respectively. Since the process of glueing makes the ordering of the $m$ glueing points contained in $\mathcal{Q}$ disappear, the point $\vartheta^{+}\left(\tau^{+}\left(\left[C^{+}\right]\right)\right)$is also fixed under the action of $\operatorname{Aut}_{\Gamma}\left(C^{+} ; \mathcal{Q}\right)$. Therefore, the point $\vartheta^{+}\left(\tau^{+}\left(\left[C^{+}\right]\right)\right)=\vartheta^{-}\left(\tau^{-}\left(\left[C^{+}\right]\right)\right)$is fixed unter the action of $\operatorname{Aut}(C)$, and we are done.

We have thus constructed a trivialization of the restricted projective bundle

$$
\begin{aligned}
\mathbb{P}\left(u_{0}\right)_{*}\left(\omega_{\mathcal{C}_{0} / \bar{H}_{g^{+}, n, m}}\right)^{\otimes n} \mid H_{g^{+}, n, m}^{\times} & \cong \mathbb{P}\left(f_{0}\right)_{*}\left(\omega_{C_{0} / k}\right)^{\otimes n} \times H_{g^{+}, n, m}^{\times} \\
& \cong \mathbb{P}^{N} \times H_{g^{+}, n, m}^{\times},
\end{aligned}
$$

which is fibrewise compatible with the natural inclusions of both projective bundles $\mathbb{P}\left(f_{0}^{+}\right)_{*}\left(\omega_{C_{0}^{+} / k}\left(Q_{1}+\cdots+Q_{m}\right)\right)^{\otimes n}$ and $\mathbb{P}\left(f_{0}^{-}\right)_{*}\left(\omega_{C_{0}^{-} / k}\left(Q_{1}+\cdots+\right.\right.$ $\left.\left.Q_{m}\right)\right)^{\otimes n}$ into $\mathbb{P}\left(f_{0}\right)_{*}\left(\omega_{C_{0} / k}\right)^{\otimes n}$, as constructed above. Globally, there is a commutative diagram of projective bundles over $H_{g^{+}, n, m}^{\times}$:

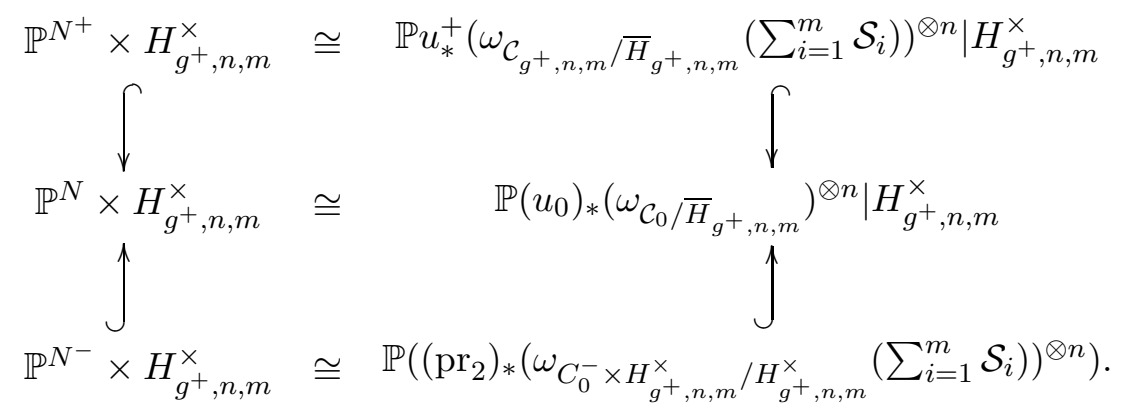

By the universal property of the Hilbert scheme $\bar{H}_{g^{+}, n, m}$, the trivialization of the projective bundle $\mathbb{P}\left(f_{0}\right)_{*}\left(\omega_{C_{0} / k}\right)^{\otimes n}$ restricted to $H_{g^{+}, n, m}^{\times}$defines a morphism $\Theta^{\times}: H_{g^{+}, n, m}^{\times} \rightarrow \bar{H}_{g, n, 0}$, which in general is not an embedding. 
By construction, this morphism is equivariant with respect to the action of the group $\operatorname{PGL}\left(N^{+}+1\right)$, considered as a subgroup of $\operatorname{PGL}(N+1)$ as in Remark 4.5.

We summarize our construction in the following proposition. Using the universal property of Hilbert schemes, it is formulated geometrically in terms of embedded curves rather than those of projective bundles, while the content is of course equivalent to our construction.

Proposition 4.11. Let $\mathcal{C}_{g^{+}, n, m}^{\times} \rightarrow H_{g^{+}, n, m}^{\times}$be the restriction of the universal embedded $m$-pointed stable curve on the Hilbert scheme $\bar{H}_{g^{+}, n, m}$ to $H_{g^{+}, n, m}^{\times}$, and let $u_{0}^{\times}: \mathcal{C}_{0}^{\times} \rightarrow H_{g^{+}, n, m}^{\times}$denote the stable curve of genus $g$ obtained by glueing it along the $m$ given sections to the trivial $m$-pointed prestable curve $C_{0}^{-} \times H_{g^{+}, n, m}^{\times} \rightarrow H_{g^{+}, n, m}^{\times}$.

Then there exists a global embedding of the curve $u_{0}^{\times}: \mathcal{C}_{0}^{\times} \rightarrow H_{g^{+}, n, m}^{\times}$ into $\mathrm{pr}_{2}: \mathbb{P}^{N} \times H_{g^{+}, n, m}^{\times} \rightarrow H_{g^{+}, n, m}^{\times}$, which makes the following diagram commutative:

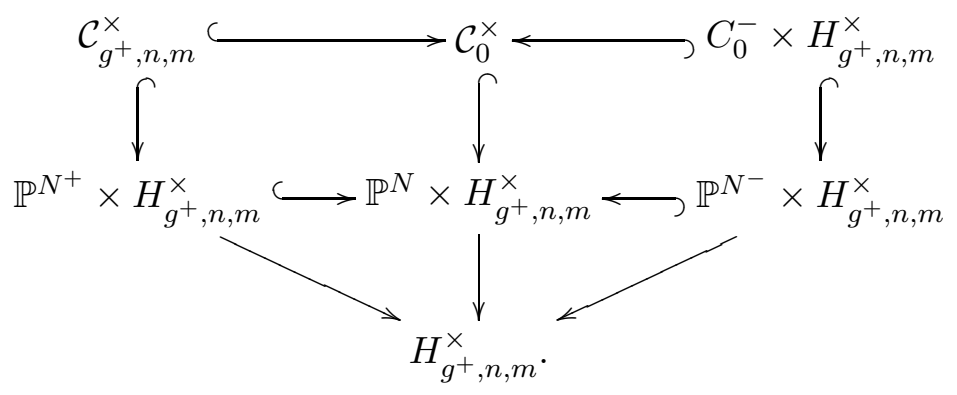

In particular, there exists a $\mathrm{PGL}\left(N^{+}+1\right)$-equivariant morphism

$$
\Theta^{\times}: H_{g^{+}, n, m}^{\times} \longrightarrow \bar{H}_{g, n, 0} .
$$

Proof. The construction was given in four steps in the paragraphs 4.4, 4.6, 4.7, and 4.10.

Proposition 4.12. The morphism $\Theta^{\times}: H_{g^{+}, n, m}^{\times} \rightarrow \bar{H}_{g, n, 0}$ from Proposition 4.11 is invariant under the action of $\Gamma\left(C_{0}^{-} ; \mathcal{Q}\right)$.

Proof. Recall that $\Gamma\left(C_{0}^{-} ; \mathcal{Q}\right)$ acts freely on $\bar{H}_{g^{+}, n, m}$. The projective bundle $\mathbb{P} u_{*}^{+}\left(\omega_{\mathcal{C}_{g^{+}, n, m}} / \bar{H}_{g^{+}, n, m}\left(\mathcal{S}_{1}+\ldots+\mathcal{S}_{m}\right)\right)^{\otimes n}$ is invariant under the action 
of $\Sigma_{m}$, and hence in particular under the action of $\Gamma\left(C_{0}^{-} ; \mathcal{Q}\right)$. This is just saying that the embedding of a fibre of $u^{+}: \mathcal{C}_{g^{+}, n, m} \rightarrow \bar{H}_{g^{+}, n, m}$ into $\mathbb{P}^{N^{+}}$ does not depend on the order of the marked points. By construction 4.4, the embedding of this projective bundle into $\mathbb{P}\left(u_{0}\right)_{*}\left(\omega_{\mathcal{C}_{0} / \bar{H}_{g^{+}, n, m}}\right)^{\otimes n}$ is also independent of the ordering of the marked points. Thus for any point $\left[C^{\prime}\right] \in$ $H_{g^{+}, n, m}^{\times}$representing an embedded curve $C^{\prime} \subset \mathbb{P}^{N^{+}}$, the embedding of $C^{\prime}$ into $\mathbb{P}^{N}$ is the same as the embedding corresponding to the point $\gamma\left(\left[C^{\prime}\right]\right)$, for any $\gamma \in \Gamma\left(C_{0}^{-} ; \mathcal{Q}\right)$. So it remains to consider the complement of $C^{\prime}$ in the curve $C$ representing the point $\Theta^{\times}\left(\left[C^{\prime}\right]\right)$. The closure of the complement of $C^{\prime}$ in $C$ is just the distinguished embedding of the curve $C_{0}^{-}$, by construction of the morphism $\Theta^{\times}$. By definition of $\Gamma\left(C_{0}^{-} ; \mathcal{Q}\right)$, and by the choice of the embedding of $\operatorname{Aut}\left(C_{0}^{-} ; \mathcal{Q}\right)$ into $\operatorname{PGL}(N+1)$ this complement is also invariant under the action of $\Gamma\left(C_{0}^{-} ; \mathcal{Q}\right)$. Therefore the whole curve $C \subset \mathbb{P}^{N}$, and hence the point $\Theta^{\times}\left(\left[C^{\prime}\right]\right) \in \bar{H}_{g, n, 0}$, is fixed under the action of $\Gamma\left(C_{0}^{-} ; \mathcal{Q}\right)$.

COROLlary 4.13. The morphism $\Theta^{\times}: H_{g^{+}, n, m}^{\times} \rightarrow \bar{H}_{g, n, 0}$ is equivariant with respect to the action of the group $\operatorname{PGL}\left(N^{+}+1\right) \times \Gamma\left(C_{0}^{-} ; \mathcal{Q}\right)$.

Proof. The equivariance with respect to the individual groups follows from Proposition 4.11 and Proposition 4.12. Note that the actions of the groups $\operatorname{PGL}\left(N^{+}+1\right)$ and $\Gamma\left(C_{0}^{-} ; \mathcal{Q}\right)$ on $H_{g^{+}, n, m}^{\times}$commute, since the embedding of an $m$-pointed stable curve into $\mathbb{P}^{N^{+}}$is independent of the ordering of its marked points.

Remark 4.14. We can view the group $\operatorname{Aut}\left(C_{0}^{-}\right) \cong \operatorname{Stab}_{\mathrm{PGL}\left(N^{-}+1\right)}\left(\left[C_{0}^{-}\right]\right)$ as a subgroup of $\operatorname{PGL}\left(N^{-}+1\right)$, and thus as a subgroup of $\operatorname{PGL}(N+1)$. The inclusion is determined by the fixed embedding of $\mathbb{P}^{N^{-}}$into $\mathbb{P}^{N}$. By definition, elements of $\operatorname{Aut}\left(C_{0}^{-}\right)$fix the $m$ marked points of $C_{0}^{-}$, which span the intersection $\mathbb{P}^{N^{-}} \cap \mathbb{P}^{N^{+}}$in $\mathbb{P}^{N}$ by Remark 4.7. Thus such an element acts as the identity on the intersection, and therefore elements of $\operatorname{Aut}\left(C_{0}^{-}\right)$ and elements of $\operatorname{PGL}\left(N^{+}+1\right)$ commute inside $\operatorname{PGL}(N+1)$. In particular, we can view $\operatorname{PGL}\left(N^{+}+1\right) \times \operatorname{Aut}\left(C_{0}^{-}\right)$as a subgroup of $\operatorname{PGL}(N+1)$.

Furthermore, the action of $\operatorname{Aut}\left(C_{0}^{-}\right)$on $\bar{H}_{g, n, 0}$ commutes with the action of $\Gamma\left(C_{0}^{-} ; \mathcal{Q}\right)$, since the embedding of $C_{0}^{-}$into $\mathbb{P}^{N}$ is independent of the labels of the $m$ marked points. We can thus strengthen Corollary 4.13 as follows.

Lemma 4.15. The morphism $\Theta^{\times}: H_{g^{+}, n, m}^{\times} \rightarrow \bar{H}_{g, n, 0}$ is equivariant with respect to the action of $\operatorname{PGL}\left(N^{+}+1\right) \times \Gamma\left(C_{0}^{-} ; \mathcal{Q}\right) \times \operatorname{Aut}\left(C_{0}^{-}\right)$, where $\operatorname{Aut}\left(C_{0}^{-}\right)$acts trivially on $H_{g^{+}, n, m}^{\times}$. 
Proof. It only remains to show that the image of $H_{g^{+}, n, m}^{\times}$under $\Theta^{\times}$is pointwise fixed under the action of $\operatorname{Aut}\left(C_{0}^{-}\right)$. Recall that $\Theta^{\times}$is constructed via compatible trivializations as in the following diagram:

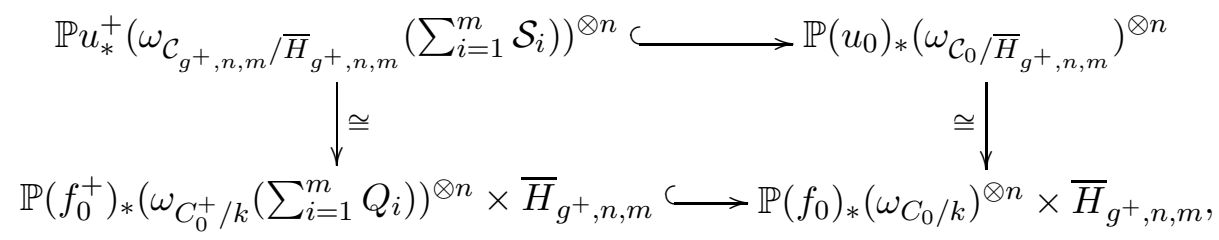

restricted to the subscheme $H_{g^{+}, n, m}^{\times}$. By definition of the inclusion of Aut $\left(C_{0}\right)$ into $\operatorname{PGL}(N+1)$, the embedding of $C_{0}$ into $\mathbb{P}^{N}$ is fixed under the action of $\operatorname{Aut}\left(C_{0}\right)$, and hence in particular fixed under the action of $\operatorname{Aut}\left(C_{0}^{-}\right)$. Therefore the embedding of the projective bundles is invariant under the action of $\operatorname{Aut}\left(C_{0}^{-}\right)$, and thus $\Theta^{\times}$is also invariant with respect to the action of $\operatorname{Aut}\left(C_{0}^{-}\right)$.

Proposition 4.16. Let $\Gamma:=\Gamma\left(C_{0}^{-} ; \mathcal{Q}\right)$. The morphism $\Theta^{\times}$induces an embedding

$$
\Theta: H_{g^{+}, n, m / \Gamma}^{\times} \longrightarrow \bar{H}_{g, n, 0}
$$

and an isomorphism of schemes $M_{g^{+}, m / \Gamma}^{\times} \cong D^{\times}\left(C_{0} ; P_{1}\right)$.

Proof. Proposition 4.12 implies that the morphism $\Theta^{\times}: H_{g^{+}, n, m}^{\times} \rightarrow$ $\bar{H}_{g, n, 0}$ factors through $H_{g^{+}, n, m / \Gamma}^{\times}=H_{g^{+}, n, m}^{\times} / \Gamma$.

Let $C_{1}$ and $C_{2}$ denote two embedded $m$-pointed stable curves over $\operatorname{Spec}(k)$, represented by two points $\left[C_{1}\right],\left[C_{2}\right] \in H_{g^{+}, n, m}^{\times}$. Suppose that $\Theta^{\times}\left(\left[C_{1}\right]\right)=\Theta^{\times}\left(\left[C_{2}\right]\right) \in \bar{H}_{g, n, 0}$, and let $C$ denote the embedded curve representing this point. By the assumption on $\left[C_{1}\right]$ and $\left[C_{2}\right]$, the curve $C$ has $3 g-4$ nodes, so there is a unique irreducible component on $C$, which has not the maximal number of nodes when considered as a pointed stable curve itself. This component is of course equal to the embedded curves $C_{1}$ and $C_{2}$ in $\mathbb{P}^{N}$, considered as curves with $m$ distinguished points, but without ordered labels on these points. The closure of its complement in $C$ is isomorphic to $C_{0}^{-}$by definition of the morphism $\Theta^{\times}$. Since $\Theta^{\times}\left(\left[C_{1}\right]\right)$ and $\Theta^{\times}\left(\left[C_{2}\right]\right)$ represent the same embedded curve, the order of the marked points of $C_{1}$ and $C_{2}$ can differ at most by a reordering induced by an automorphism of $C_{0}^{-}$. Hence by definition of $\Gamma\left(C_{0}^{-} ; \mathcal{Q}\right)$, the points $\left[C_{1}\right]$ and $\left[C_{2}\right]$ are the same modulo the action of $\Gamma\left(C_{0}^{-} ; \mathcal{Q}\right)$. This proves the first part of the proposition. 
For the second part note that by Corollary 4.13 the morphism $\Theta^{\times}$induces a morphism $M_{g^{+}, m / \Gamma}^{\times} \rightarrow \bar{M}_{g}$. By the definition of $M_{g^{+}, m}^{\times}$, its image is $D^{\times}\left(C_{0} ; P_{1}\right)$. The morphism is just the restriction of the isomorphism from Lemma 3.4.

\section{§5. Decomposition of stable curves}

Notation 5.1. Let $\pi: \bar{H}_{g, n, 0} \rightarrow \bar{M}_{g}$ be the canonical quotient morphism. We denote by

$$
K\left(C_{0} ; P_{1}\right) \subset \bar{H}_{g, n, 0}
$$

the reduction of the preimage of $D\left(C_{0} ; P_{1}\right)$. Furthermore, we denote by $K^{\times}\left(C_{0} ; P_{1}\right)$ the reduction of the preimage of the open subscheme $D^{\times}\left(C_{0} ; P_{1}\right)$, which represents curves with splitting automorphism groups.

LEMmA 5.2. The subscheme $K^{\times}\left(C_{0} ; P_{1}\right)$ is equal to the reduction of the $\operatorname{PGL}(N+1)$-orbit $K^{\times}\left(C_{0} ; P_{1}\right)=\Theta^{\times}\left(H_{g^{+}, n, m}^{\times}\right) \cdot \operatorname{PGL}(N+1)$ of the image of the morphism $\Theta^{\times}: H_{g^{+}, n, m}^{\times} \rightarrow \bar{H}_{g, n, 0}$ from Proposition 4.11 .

Proof. This follows immediately from the definition of $K^{\times}\left(C_{0} ; P_{1}\right)$, and the surjectivity of the morphism $M_{g^{+}, m}^{\prime} \rightarrow D\left(C_{0} ; P_{1}\right)$.

Remark 5.3. The scheme $K\left(C_{0} ; P_{1}\right)$ is a smooth and irreducible subscheme of $\bar{H}_{g, n, 0}$. This follows from the definition of $D\left(C_{0} ; P_{1}\right)$ as a connected component of $M_{g}^{(3 g-4)}$, and the fact that the boundary $\bar{H}_{g, n, 0} \backslash H_{g, n, 0}$ is a normal crossing divisor, compare [DM].

LEMmA 5.4. Let $f: C \rightarrow S$ be a stable curve of genus $g$, with reduced and irreducible base $S$, such that for all closed points $s \in S$ the fibre $C_{s}$ has exactly $3 g-4$ nodes. Then the induced morphism $\vartheta_{f}: S \rightarrow \bar{M}_{g}$ factors through $D\left(C_{0} ; P_{1}\right)$ if and only if there exists an étale covering $u: S^{\prime} \rightarrow S$, such that the pullback stable curve $f^{\prime}: C^{\prime} \rightarrow S^{\prime}$ decomposes as follows. There is an m-pointed prestable subcurve, which is unique up to permutations of the labels of its marked points, and isomorphic to the trivial curve $C_{0}^{-} \times S^{\prime} \rightarrow S^{\prime}$, together with an m-pointed stable curve $f^{\#}: C^{\#} \rightarrow S^{\prime}$ of genus $g^{+}$, such that the curve $f^{\prime}: C^{\prime} \rightarrow S^{\prime}$ is obtained by glueing the two subcurves along the corresponding sections of marked points.

Proof. Suppose that such an étale covering exists. Since $f^{\prime}: C^{\prime} \rightarrow S^{\prime}$ is the pullback of $f: C \rightarrow S$, it too is a stable curve of genus $g$ with exactly 
$3 g-4$ nodes in each fibre, and the induced morphism $\vartheta_{f^{\prime}}: S^{\prime} \rightarrow \bar{M}_{g}$ factors as $\vartheta_{f^{\prime}}=\vartheta_{f} \circ u$. Thus $\vartheta_{f}$ and $\vartheta_{f^{\prime}}$ map to the same irreducible component $D$ of $M_{g}^{(3 g-4)}$, since $S$ is irreducible. For a general point $s \in S^{\prime}$, the closure of the complement of $C_{0}^{-}$in the fibre $C_{s}^{\prime}$ is an $m$-pointed stable curve of genus $g^{+}$. Thus by definition $\vartheta_{f^{\prime}}(s) \in D\left(C_{0} ; P_{1}\right)$, and hence $D=D\left(C_{0} ; P_{1}\right)$.

Conversely, suppose that $\vartheta_{f}: S \rightarrow \bar{M}_{g}$ factors through $D\left(C_{0} ; P_{1}\right)$. By Lemma 2.7 there exists an étale covering $u: S^{\prime} \rightarrow S$, such that $S^{\prime}$ is connected, and the pullback $f^{\prime}: C^{\prime} \rightarrow S^{\prime}$ admits $3 g-4$ sections of nodes. For a closed point $s \in S^{\prime}$ there are two types of nodes on the fibre $C_{s}^{\prime}$ : those nodes, which are intersection points of different irreducible components of $C_{s}^{\prime}$, and those which are singularities of one irreducible component. Since the curve $f^{\prime}: C^{\prime} \rightarrow S^{\prime}$ is locally a deformation of its fibre $C_{s}^{\prime}$, and since the number of nodes is constant for all fibres, all nodes of one section must be of the same type.

Suppose that $\sigma_{1}, \ldots, \sigma_{k}: S^{\prime} \rightarrow C^{\prime}$ are the sections of nodes, which are intersection points. Let $\tilde{f}: \tilde{C} \rightarrow S^{\prime}$ denote the flat family, which is obtained by "cutting" the curve $C^{\prime}$ along this $k$ sections. Formally, we may construct $\tilde{C}$ by choosing an embedding of $f^{\prime}: C^{\prime} \rightarrow S^{\prime}$ into $\mathbb{P}^{\ell} \times S^{\prime} \rightarrow S^{\prime}$, for some sufficiently large $\ell$, and blowing up along the subschemes $\sigma_{1}\left(S^{\prime}\right), \ldots, \sigma_{k}\left(S^{\prime}\right)$. The family $\tilde{f}: \tilde{C} \rightarrow S^{\prime}$ is then given as the strict transform of $f^{\prime}: C^{\prime} \rightarrow S^{\prime}$, where the points of $C^{\prime}$ contained in the $k$ sections are separated into pairs of points of $\tilde{C}$ above.

Note that $\tilde{f}: \tilde{C} \rightarrow S^{\prime}$ is a pointed prestable curve over $S^{\prime}$. In particular, since $S^{\prime}$ is connected, for any connected component of $Z \subset \tilde{C}$ the restriction $\tilde{f} \mid Z: Z \rightarrow S^{\prime}$ is still a pointed stable curve. Thus the genus and the number of nodes remains constant for all fibres of $\tilde{f} \mid Z$. For a general closed point $s \in S^{\prime}$, the fibre $C_{s}^{\prime}$ has exactly $3 g-4$ nodes. Hence there exists a unique irreducible component of $C_{s}^{\prime}$, which has not the maximal number of nodes when considered as a pointed stable curve itself. Therefore there exists a unique connected component of the fibre $\tilde{C}_{s}$, which has not the maximal number of nodes when considered as a pointed stable curve. This component is necessarily an $m$-pointed stable curve of genus $g^{+}$. This implies that there exists a unique connected component $C^{\#}$ of $\tilde{C}$, which is an $m$-pointed stable curve of genus $g^{+}$over $S^{\prime}$. By construction, this curve $f^{\#}: C^{\#} \rightarrow S^{\prime}$ can be considered as a subcurve embedded in $f^{\prime}: C^{\prime} \rightarrow S^{\prime}$.

Let $f^{-}: C^{-} \rightarrow S^{\prime}$ denote the family obtained as the closure of the complement of $f^{\#}: C^{\#} \rightarrow S^{\prime}$ in $f^{\prime}: C^{\prime} \rightarrow S^{\prime}$. Let $s \in S^{\prime}$ be a closed point, such that $\vartheta_{f^{\prime}}(s) \in D\left(C_{0} ; P_{1}\right)$. Then the fibre $C_{s}^{\prime}$ must contain a subcurve 
isomorphic to $C_{0}^{-}$by definition. All irreducible components of $C_{0}^{-}$have the maximal number of nodes when considered as pointed stable curves, so $C_{0}^{-}$ must be equal to the closure of the complement of $C_{s}^{\#}$ in $C_{s}^{\prime}$. Thus, all fibres of the $m$-pointed prestable curve $f^{-}: C^{-} \rightarrow S^{\prime}$ are isomorphic to $C_{0}^{-}$. In particular, after applying another étale covering of $S^{\prime}$ if necessary, the curve $f^{-}: C^{-} \rightarrow S^{\prime}$ is isomorphic to the trivial family $C_{0}^{-} \times S^{\prime} \rightarrow S^{\prime}$.

Note that although $f^{\#}: C^{\#} \rightarrow S^{\prime}$ is uniquely determined as a subscheme, being the complement of $C_{0}^{-} \times S^{\prime} \rightarrow S^{\prime}$, the labels of its $m$ sections of marked points depend on choices made during the process of glueing.

Corollary 5.5. Let $f: C \rightarrow S$ be a stable curve of genus $g$, with reduced base $S$. Suppose that the induced morphism $\vartheta_{f}: S \rightarrow \bar{M}_{g}$ factors through $D\left(C_{0} ; P_{1}\right)$. Then there exists a uniquely determined decomposition of $f: C \rightarrow S$ into an $m / \Gamma\left(C_{0}^{-} ; \mathcal{Q}\right)$-pointed stable curve $f^{+}: C^{+} \rightarrow S$ of genus $g^{+}$, and an $m / \Gamma\left(C_{0}^{-} ; \mathcal{Q}\right)$-pointed prestable curve $f^{-}: C^{-} \rightarrow S$ of genus $g^{-}$, where the second curve is locally isomorphic to the trivial curve $C_{0}^{-} \times S \rightarrow S$ with respect to the étale topology.

Proof. Without loss of generality we may assume that $S$ is irreducible. Indeed, if decompositions exist on the irreducible components of $S$, then the uniqueness property implies that they fit together to a global decomposition.

By Lemma 5.4 there exists an étale covering $u: S^{\prime} \rightarrow S$, such that the pullback curve $f^{\prime}: C^{\prime} \rightarrow S^{\prime}$ decomposes into an $m$-pointed stable curve $f^{\#}: C^{\#} \rightarrow S^{\prime}$, and a trivial $m$-pointed prestable curve $C_{0}^{-} \times S^{\prime} \rightarrow S^{\prime}$. By assumption, each fibre of $f: C \rightarrow S$ has exactly $3 g-4$ nodes. Consider the pullback morphism $\bar{u}: C^{\prime} \rightarrow C$. For a closed point $s \in S^{\prime}$, it maps the fibre $C_{s}^{\#}$ of the subcurve $f^{\#}: C^{\#} \rightarrow S^{\prime}$ isomorphically onto the unique irreducible component of the fibre $C_{u(s)}$, which has not the maximal number of nodes when considered as a pointed stable curve. In particular, the image of $C^{\#}$ in $C$ defines uniquely a subfamily $f^{+}: C^{+} \rightarrow S$ in $f: C \rightarrow S$.

Analogously, the image of the trivial subcurve $C_{0}^{-} \times S^{\prime} \rightarrow S^{\prime}$ defines a subfamily $f^{-}: C^{-} \rightarrow S$. Since the latter is the étale image of an $m$-pointed prestable curve, it is by definition an $m / \Gamma\left(C_{0}^{-} ; \mathcal{Q}\right)$-pointed curve.

Let $s, s^{\prime} \in S^{\prime}$ be two closed points with $u(s)=u\left(s^{\prime}\right)$. If $C_{s}^{\#}$ and $C_{s^{\prime}}^{\#}$ denote the two corresponding fibres of $f^{\#}: C^{\#} \rightarrow S^{\prime}$, then glueing in the $m$ marked points to $C_{0}^{-}$produces in both cases the same fibre $C_{u(s)}=C_{u\left(s^{\prime}\right)}$ of $f: C \rightarrow S$. Thus the labels of the respective $m$ marked points may 
differ only by a permutation induced by an automorphisms of $C_{0}^{-}$, i.e. by a permutation contained in $\Gamma\left(C_{0}^{-} ; \mathcal{Q}\right)$. Thus the induced curve $f^{+}: C^{+} \rightarrow S$ is an $m / \Gamma\left(C_{0}^{-} ; \mathcal{Q}\right)$-pointed stable curve of genus $g^{+}$, as defined in [Z2].

The above Corollary 5.5 deals only with stable curves $f: C \rightarrow S$, where the base $S$ is reduced. In our applications, we will need an analogous statement for arbitrary schemes $S$, and for this we need one extra assumption.

Remark 5.6. There is a functorial way to assign to each pointed stable curve $f: C \rightarrow S$ a pair $\left(E_{C / S}, \phi_{C / S}\right)$, consisting of a principal bundle $p_{C / S}: E_{C / S} \rightarrow S$, and an equivariant morphism $\phi_{C / S}: E_{C / S} \rightarrow \bar{H}_{g, n, m}$. We recall briefly the description of this construction from [Z2].

Let $f: C \rightarrow S$ be an $m$-pointed stable curve of genus $g$, with sections of marked points $\sigma_{1}, \ldots, \sigma_{m}: S \rightarrow C$. The sections define divisors $S_{1}, \ldots, S_{m}$ on $C$. We denote by $p_{C / S}: E_{C / S} \rightarrow S$ the $\operatorname{PGL}(N+1)$-principal bundle associated to the projective bundle $P:=\mathbb{P} f_{*}\left(\omega_{C / S}\left(S_{1}+\cdots+S_{m}\right)\right)^{\otimes n}$ over $S$. The pullback of $P$ to $E_{C / S}$ has a natural trivialization as a projective bundle. This implies that the pullback stable curve $\bar{f}: \bar{C} \rightarrow E_{C / S}$ of the stable curve $f: C \rightarrow S$ has a natural embedding into $\mathbb{P}^{N} \times E_{C / S}$. By the universal property of the Hilbert scheme this is equivalent to specifying a $\operatorname{PGL}(N+1)$-equivariant morphism $\phi_{C / S}: E_{C / S} \rightarrow \bar{H}_{g, n, m}$.

It is straightforward to see that this construction is functorial. The assignment is even reversible: let $u: \mathcal{C}_{g, n, m} \rightarrow \bar{H}_{g, n, m}$ denote the universal curve. Then the curve $f: C \rightarrow S$ can be recovered from the pair $\left(E_{C / S}, \phi_{C / S}\right)$ by taking the quotient $\phi_{C / S}^{*}\left(\mathcal{C}_{g, n, m}\right) / \operatorname{PGL}(N+1) \rightarrow$ $E_{C / S} / \operatorname{PGL}(N+1)$.

Proposition 5.7. Let $f: C \rightarrow S$ be a stable curve of genus $g$. Suppose that the induced morphism $\vartheta_{f}: S \rightarrow \bar{M}_{g}$ factors through $D\left(C_{0} ; P_{1}\right)$. Let $\left(E_{C / S}, \phi_{C / S}\right)$ be the pair associated to $f: C \rightarrow S$ as in Remark 5.6. Suppose that $\phi_{C / S}$ factors through $K\left(C_{0} ; P_{1}\right)$. Then there exists a uniquely determined decomposition of $f: C \rightarrow S$ into an $m / \Gamma\left(C_{0}^{-} ; \mathcal{Q}\right)$-pointed stable curve $f^{+}: C^{+} \rightarrow S$ of genus $g^{+}$, and an $m / \Gamma\left(C_{0}^{-} ; \mathcal{Q}\right)$-pointed prestable curve $f^{-}: C^{-} \rightarrow S$ of genus $g^{-}$, where the second curve is locally isomorphic to the trivial curve $C_{0}^{-} \times S \rightarrow S$ with respect to the étale topology.

Proof. Let $\mathcal{C}_{g, n, 0} \rightarrow \bar{H}_{g, n, 0}$ denote the universal curve, and $\mathcal{C}_{K} \rightarrow$ $K\left(C_{0} ; P_{1}\right)$ its restriction to $K\left(C_{0} ; P_{1}\right)$. Since $K\left(C_{0} ; P_{1}\right)$ is reduced, Corollary 5.5 shows the existence of a unique subcurve $\mathcal{C}_{K}^{-}$, which is locally isomorphic 
to the trivial curve $C_{0}^{-} \times K\left(C_{0} ; P_{1}\right)$ as an $m / \Gamma\left(C_{0}^{-} ; \mathcal{Q}\right)$-pointed curve. Let $\mathcal{C}_{E}$ and $\mathcal{C}_{E}^{-}$denote the respective pullbacks of $\mathcal{C}_{K}$ and $\mathcal{C}_{K}^{-}$to $E_{C / S}$. By the definition of $E_{C / S}$, one has $f: C=\mathcal{C}_{E} / \operatorname{PGL}(N+1) \rightarrow E_{C / S} / \operatorname{PGL}(N+1)=S$, and $C^{-}:=\mathcal{C}_{E}^{-} / \operatorname{PGL}(N+1) \rightarrow S$ is the unique subcurve, which is locally isomorphic to $C_{0}^{-} \times S \rightarrow S$ as an $m / \Gamma\left(C_{0}^{-} ; \mathcal{Q}\right)$-pointed curve.

\section{§6. The moduli substack}

After our discussion on the level of moduli spaces, let us now define the corresponding substacks in the boundary of the moduli stack $\overline{\mathcal{M}}_{g}$ of Deligne-Mumford stable curves of genus $g$.

Definition 6.1. Let $\tilde{\mathcal{D}}\left(C_{0} ; P_{1}\right)$ denote the preimage substack of the scheme $D\left(C_{0} ; P_{1}\right)$ in $\overline{\mathcal{M}}_{g}$ under the canonical map from $\overline{\mathcal{M}}_{g}$ to $\bar{M}_{g}$. The stack $\mathcal{D}\left(C_{0} ; P_{1}\right)$ is defined as the reduced stack underlying $\tilde{\mathcal{D}}\left(C_{0} ; P_{1}\right)$. We define the substack $\mathcal{D}^{\times}\left(C_{0} ; P_{1}\right)$ as the reduction of the preimage of the open and dense subscheme $D^{\times}\left(C_{0} ; P_{1}\right)$.

Remark 6.2. For any scheme $S$, the set of objects of the fibre category $\tilde{\mathcal{D}}\left(C_{0} ; P_{1}\right)(S)$ is equal to the set of those stable curves $f: C \rightarrow S$ of genus $g$, such that the induced morphism $\vartheta_{f}: S \rightarrow \bar{M}_{g}$ factors through the subscheme $D\left(C_{0} ; P_{1}\right)$. It follows from Proposition 5.7 that $\mathcal{D}\left(C_{0} ; P_{1}\right)$ is a moduli stack for curves $f: C \rightarrow \operatorname{Spec}(k)$, which contain a subcurve isomorphic to $C_{0}^{-}$, where the marked points on $C_{0}^{-}$correspond to nodes on $C$, and that $D\left(C_{0} ; P_{1}\right)$ is its moduli space.

LEMMA 6.3. There are isomorphisms of stacks

$$
\mathcal{D}\left(C_{0} ; P_{1}\right) \cong\left[K\left(C_{0} ; P_{1}\right) / \operatorname{PGL}(N+1)\right],
$$

and

$$
\mathcal{D}^{\times}\left(C_{0} ; P_{1}\right) \cong\left[K^{\times}\left(C_{0} ; P_{1}\right) / \operatorname{PGL}(N+1)\right] .
$$

In particular, both stacks are smooth and irreducible, and of dimension one.

Proof. It was shown by Edidin [E] that the moduli stack of stable curves of genus $g$ can be constructed as a quotient stack $\overline{\mathcal{M}}_{g} \cong\left[\bar{H}_{g, n, 0} / \operatorname{PGL}(N+\right.$ $1)$. Using this, the descriptions of $\mathcal{D}\left(C_{0} ; P_{1}\right)$ and $\mathcal{D}^{\times}\left(C_{0} ; P_{1}\right)$ follow immediately from the definitions by standard arguments on Cartesian products and quotient stacks. Since by Remark 5.3 the scheme $K\left(C_{0} ; P_{1}\right)$ is smooth and irreducible as an atlas of $\mathcal{D}\left(C_{0} ; P_{1}\right)$, so is $\mathcal{D}\left(C_{0} ; P_{1}\right)$ itself. 
Remark 6.4. From Lemma 6.3 the following characterization of $\mathcal{D}\left(C_{0} ; P_{1}\right)$ can be derived. For any scheme $S$, the fibre category $\mathcal{D}\left(C_{0} ; P_{1}\right)(S)$ has as its objects such stable curves $f: C \rightarrow S$ of genus $g$, where the induced morphism $\vartheta_{f}: S \rightarrow \bar{M}_{g}$ factors through $D\left(C_{0} ; P_{1}\right)$, and the morphism $\phi_{C / S}: E_{C / S} \rightarrow \bar{H}_{g, n, 0}$ from Remark 5.6 factors through $K\left(C_{0} ; P_{1}\right)$.

Notation 6.5. Let $\mathcal{M}_{g^{+}, m}^{\prime}$ denote the substack of $\overline{\mathcal{M}}_{g^{+}, m}$ which is the reduction of the preimage of $M_{g^{+}, m}^{\prime}$. Analogously, let $\mathcal{M}_{g^{+}, m / \Gamma}^{\prime}$ denote the substack of $\mathcal{M}_{g^{+}, m / \Gamma}^{\prime}$ which is the reduction of the preimage of $M_{g^{+}, m / \Gamma}^{\prime}$. As in 2.5 , there is a natural morphism $\vartheta: \mathcal{M}_{g^{+}, m}^{\prime} \rightarrow \overline{\mathcal{M}}_{g}$, which is given by glueing the trivial curve with fibre $C_{0}^{-}$to any $m$-pointed stable curve.

However, unlike the situation in the case of moduli spaces as described in Lemma 3.4, there is no induced isomorphism between the moduli stacks $\mathcal{M}_{g^{+}, m / \Gamma}^{\prime}$ and $\mathcal{D}\left(C_{0} ; P_{1}\right)$, where $\Gamma=\Gamma\left(C_{0}^{-} ; \mathcal{Q}\right)$. It turns out that in order to relate the two stacks, we need to consider a finite quotient of $\mathcal{M}_{g^{+}, m / \Gamma}^{\prime}$, see Remark 6.7. Even then, there is no global morphism from this quotient to $\mathcal{D}\left(C_{0} ; P_{1}\right)$, so we must furthermore restrict ourselves to the following open and dense substack.

Let us denote by $\mathcal{M}_{g^{+}, m / \Gamma}^{\times}$the substack of $\overline{\mathcal{M}}_{g^{+}, m / \Gamma}$, which is the reduction of the preimage of $M_{g^{+}, m / \Gamma}^{\times}$. Analogously we define $\mathcal{M}_{g^{+}, m}^{\times}$in $\overline{\mathcal{M}}_{g^{+}, m}$.

By Lemma 4.15 and Proposition 4.16, there exists a $\operatorname{PGL}\left(N^{+}+1\right) \times$ $\operatorname{Aut}\left(C_{0}^{-}\right)$-equivariant morphism $\Theta: H_{g^{+}, n, m / \Gamma}^{\times} \rightarrow \bar{H}_{g, n, 0}$, where $\operatorname{Aut}\left(C_{0}^{-}\right)$ acts trivially on $H_{g^{+}, n, m / \Gamma^{\circ}}$. The following proposition provides a stack version of the induced morphism $M_{g^{+}, m / \Gamma}^{\times} \rightarrow D^{\times}\left(C_{0} ; P_{1}\right)$.

Proposition 6.6. There is a morphism of Deligne-Mumford stacks

$$
\Lambda:\left[H_{g^{+}, n, m / \Gamma}^{\times} / \operatorname{PGL}\left(N^{+}+1\right) \times \operatorname{Aut}\left(C_{0}^{-}\right)\right] \longrightarrow \mathcal{D}^{\times}\left(C_{0} ; P_{1}\right)
$$

where $\operatorname{Aut}\left(C_{0}^{-}\right)$acts trivially on $H_{g^{+}, n, m / \Gamma}^{\times}$and $\Gamma:=\Gamma\left(C_{0}^{-} ; \mathcal{Q}\right)$.

As abbreviations, we will from now on use $A:=\operatorname{Aut}\left(C_{0}^{-}\right), \Gamma:=$ $\Gamma\left(C_{0}^{-} ; \mathcal{Q}\right)$ and $P:=\operatorname{PGL}\left(N^{+}+1\right)$. We will also denote the subschemes $K^{\times}\left(C_{0} ; P_{1}\right)$ and $D^{\times}\left(C_{0} ; P_{1}\right)$ simply by $K^{\times}$and $D^{\times}$, respectively.

Remark 6.7. In [Z2], the moduli stack of $m / \Gamma$-pointed stable curves has been constructed as a quotient stack $\overline{\mathcal{M}}_{g^{+}, m / \Gamma} \cong\left[\bar{H}_{g^{+}, n, m / \Gamma} / P\right]$. Thus 
there is also an isomorphism $\mathcal{M}_{g^{+}, m / \Gamma}^{\times} \cong\left[H_{g^{+}, n, m / \Gamma}^{\times} / P\right]$. By composing the morphism $\Lambda$ of Proposition 6.6 with the canonical quotient morphism $\mathcal{M}_{g^{+}, m / \Gamma}^{\times} \rightarrow \mathcal{M}_{g^{+}, m / \Gamma}^{\times} / A=\left[H_{g^{+}, n, m / \Gamma}^{\times} / P \times A\right]$, we obtain a morphism of stacks $\mathcal{M}_{g^{+}, m / \Gamma}^{\times} \rightarrow \overline{\mathcal{M}}_{g}$, which factors through $\mathcal{D}^{\times}\left(C_{0} ; P_{1}\right)$, and which induces the natural morphism $M_{g^{+}, m}^{\times} \rightarrow D^{\times}\left(C_{0} ; P_{1}\right)$ on the corresponding moduli spaces.

Proof of Proposition 6.6. Viewing stacks as categories fibred in groupoids over the category of schemes, the morphism $\Lambda$ will be given as a functor respecting the fibrations. Let a scheme $S$ be fixed. Recall that an object of $\left[H_{g^{+}, n, m / \Gamma}^{\times} / P \times A\right](S)$ is a triple $\left(E^{\prime}, p^{\prime}, \phi^{\prime}\right)$, where $p^{\prime}: E^{\prime} \rightarrow S$ is a principal $P \times A$-bundle, together with a $P \times A$-equivariant morphism $\phi^{\prime}$ : $E^{\prime} \rightarrow H_{g^{+}, n, m / \Gamma^{\circ}}^{\times}$Note that this data is equivalent to specifying a stable curve $f: C \rightarrow S$ as an object of $\mathcal{M}_{g^{+}, m / \Gamma}^{\times}$, with $p_{C / S}=p^{\prime}$ and $\phi_{C / S}=\phi^{\prime}$, compare Remark 5.6.

Viewing $P \times A$ as a subgroup of $\operatorname{PGL}(N+1)$ as in Remark 4.14, we can define an extension $E$ of $E^{\prime}$ by

$$
E:=\left(E^{\prime} \times \operatorname{PGL}(N+1)\right) /(P \times A)
$$

as a principal PGL $(N+1)$-bundle $p: E \rightarrow S$ over $S$. By Lemma 5.2 we have $K^{\times}=\Theta^{\times}\left(H_{g^{+}, n, m}^{\times}\right) \cdot \operatorname{PGL}(N+1)=\Theta\left(H_{g^{+}, n, m / \Gamma}^{\times}\right) \cdot \operatorname{PGL}(N+1)$. Therefore we can define a morphism

$$
\begin{aligned}
\phi: E & \longrightarrow K^{\times} \\
e & \longmapsto \Theta\left(\phi^{\prime}\left(e^{\prime}\right)\right) \cdot \gamma,
\end{aligned}
$$

where $e \in E$ is represented by a pair $\left(e^{\prime}, \gamma\right) \in E^{\prime} \times \operatorname{PGL}(N+1)$. This map is indeed well-defined, as $\Theta \circ \phi^{\prime}$ is equivariant with respect to $P \times A$. For all $g \in \operatorname{PGL}(N+1)$ we have $\phi(e g)=\phi(e) g$ for all $e \in E$, so $\phi$ is $\operatorname{PGL}(N+1)$ equivariant. Hence $(E, p, \phi) \in\left[K^{\times} / \operatorname{PGL}(N+1)\right](S) \cong \mathcal{D}^{\times}\left(C_{0} ; P_{1}\right)(S)$, where the last isomorphism holds by Lemma 6.3 .

It is easy to see that this construction of extending principal bundles is functorial. In this way we obtain for each scheme $S$ a functor between the fibre categories $\Lambda_{S}:\left[H_{g^{+}, n, m / \Gamma}^{\times} / P \times A\right](S) \rightarrow \mathcal{D}^{\times}\left(C_{0} ; P_{1}\right)(S)$. This in turn defines the desired morphism of stacks $\Lambda:\left[H_{g^{+}, n, m / \Gamma}^{\times} / P \times A\right] \rightarrow \mathcal{D}^{\times}\left(C_{0} ; P_{1}\right)$ in the usual way.

We now arrive at our central result on the one-dimensional boundary strata of the moduli stack of Deligne-Mumford stable curves. 
THEOREM 6.8. There is an isomorphism of Deligne-Mumford stacks

$$
\left[H_{g^{+}, n, m / \Gamma}^{\times} / \operatorname{PGL}\left(N^{+}+1\right) \times \operatorname{Aut}\left(C_{0}^{-}\right)\right] \cong \mathcal{D}^{\times}\left(C_{0} ; P_{1}\right)
$$

where $\operatorname{Aut}\left(C_{0}^{-}\right)$acts trivially on $H_{g^{+}, n, m / \Gamma}^{\times}$, and $\Gamma:=\Gamma\left(C_{0}^{-} ; \mathcal{Q}\right)$.

Remark 6.9. ( $i$ ) By the construction given in [Z2], there is an isomorphism of stacks $\mathcal{M}_{g^{+}, m / \Gamma}^{\times} \cong\left[H_{g^{+}, n, m / \Gamma}^{\times} / \operatorname{PGL}\left(N^{+}+1\right)\right]$. Therefore Theorem 6.8 implies that generically the moduli substack $\mathcal{D}\left(C_{0} ; P_{1}\right)$ is isomorphic to the stack quotient $\mathcal{M}_{g^{+}, m / \Gamma}^{\prime} / \operatorname{Aut}\left(C_{0}^{-}\right)$. Note that since the action of $\operatorname{Aut}\left(C_{0}^{-}\right)$on $H_{g^{+}, n, m / \Gamma}^{\prime}$ is trivial, this quotient is isomorphic to the product of the moduli stack $\mathcal{M}_{g^{+}, m / \Gamma}^{\prime}$ with the classifying stack $\operatorname{BAut}\left(C_{0}^{-}\right)$ of the group $\operatorname{Aut}\left(C_{0}^{-}\right)$. In particular, even though there is an isomorphism $D^{\times}\left(C_{0} ; P_{1}\right) \cong M_{g^{+}, m / \Gamma}^{\times}$of the respective moduli spaces, the stacks $\mathcal{D}^{\times}\left(C_{0} ; P_{1}\right)$ and $\mathcal{M}_{g^{+}, m / \Gamma}^{\prime}$ are in general not isomorphic.

(ii) Theorem 6.8 is in fact the strongest result possible. Indeed, consider a stable curve $f: C \rightarrow \operatorname{Spec}(k)$, which is an object of $\mathcal{D}\left(C_{0} ; P_{1}\right)(\operatorname{Spec}(k))$, but not of $\mathcal{D}^{\times}\left(C_{0} ; P_{1}\right)(\operatorname{Spec}(k))$. An extension of the isomorphism must fail, since the splitting of the stack into a product would imply a corresponding splitting of the automorphism group $\operatorname{Aut}(C)$, as explained in [Z1], and thus contradict the definition of $\mathcal{D}^{\times}\left(C_{0} ; P_{1}\right)$.

However, Theorem 6.8 can be extended to a certain degree to the closure of $\mathcal{D}\left(C_{0} ; P_{1}\right)$. Recall that $\mathcal{D}\left(C_{0} ; P_{1}\right)$ is defined by a connected component of the boundary stratum $M_{g}^{(3 g-4)}$ parameterizing stable curves with exactly $3 g-4$ nodes. So its closure contains stable curves with at least $3 g-4$ nodes. For a full discussion and the construction of this extension, see [Z1].

Proof of Theorem 6.8. The proof is by explicit construction of an inverse morphism $\Xi$ to the morphism $\Lambda$ from Proposition 6.6 in such a way, that the composition $\Lambda \circ \Xi$ is isomorphic to, and the composition $\Xi \circ \Lambda$ is equal to the respective identity functor. As before, we are using the abbreviations $A:=\operatorname{Aut}\left(C_{0}^{-}\right), \Gamma:=\Gamma\left(C_{0}^{-} ; \mathcal{Q}\right)$ and $P:=\operatorname{PGL}\left(N^{+}+1\right)$, as well as $K^{\times}:=K^{\times}\left(C_{0} ; P_{1}\right)$ and $D^{\times}:=D^{\times}\left(C_{0} ; P_{1}\right)$.

We need to construct for each object of $\mathcal{D}^{\times}\left(C_{0} ; P_{1}\right)(S)$, for each scheme $S$, its image in $\left[H_{g^{+}, n, m / \Gamma}^{\times} / \operatorname{PGL}\left(N^{+}+1\right) \times \operatorname{Aut}\left(C_{0}^{-}\right)\right](S)$ under $\Xi$. By Lemma 6.3 we may assume that such an object is given by a triple $(E, p, \phi)$, where $p: E \rightarrow S$ is a principal $\operatorname{PGL}(N+1)$-bundle, together with a 
$\operatorname{PGL}(N+1)$-equivariant morphism $\phi: E \rightarrow K^{\times}$. In a first step we will construct from this data a principal $P \times A$-bundle $p^{+}: E^{+} \rightarrow S$.

(i) Consider the restriction $\mathcal{C}_{g, n, 0} \rightarrow K^{\times}$of the universal stable curve over $\bar{H}_{g, n, 0}$ to $K^{\times}$, and denote its pullback to $E$ via $\phi$ by $\mathcal{C}_{E} \rightarrow E$. Taking quotients by the action of $\operatorname{PGL}(N+1)$ induces a morphism

$$
f_{S}: \mathcal{C}_{S}:=\mathcal{C}_{E} / \operatorname{PGL}(N+1) \longrightarrow E / \operatorname{PGL}(N+1)=S,
$$

which is a stable curve of genus $g$ over $S$. The induced morphism $\vartheta_{f}$ : $S \rightarrow \bar{M}_{g}$ factors through $D^{\times}$. Therefore, by Proposition 5.7, there exists a unique subcurve $f_{S}^{-}: \mathcal{C}_{S}^{-} \rightarrow S$ of $f_{S}: \mathcal{C}_{S} \rightarrow S$, and an étale covering $u: S^{\prime} \rightarrow S$, such that the pullback $f_{S^{\prime}}^{-}: \mathcal{C}_{S^{\prime}}^{-} \rightarrow S^{\prime}$ of $f_{S}^{-}$is isomorphic to the trivial $m$-pointed stable curve $C_{0}^{-} \times S^{\prime} \rightarrow S^{\prime}$. Let $f_{S^{\prime}}: \mathcal{C}_{S^{\prime}} \rightarrow S^{\prime}$ denote the pullback of $f_{S}$.

Recall that in general $C_{0}^{-}$is not a stable curve, but only prestable. Suppose that $C_{0}^{-}$decomposes into connected components $C_{1}^{-}, \ldots, C_{r}^{-}$, where $C_{i}^{-}$is an $m_{i}$-pointed stable curve of some genus $g_{i}^{-}$for $i=1, \ldots, r$. This induces a decomposition of $f_{S^{\prime}}^{-}: \mathcal{C}_{S^{\prime}}^{-} \rightarrow S^{\prime}$ into subcurves $f_{S^{\prime}, i}^{-}: \mathcal{C}_{S^{\prime}, i}^{-} \rightarrow S^{\prime}$. Let $S_{1, i}^{\prime}, \ldots, S_{m_{i}, i}^{\prime}$ denote the respective sections of the $m_{i}$ marked points on $\mathcal{C}_{S^{\prime}, i}^{-}$. As in construction 4.4 there is a natural inclusion of projective bundles $\mathbb{P}\left(f_{S^{\prime}, i}^{-}\right)_{*}\left(\omega_{\mathcal{C}_{S^{\prime}, i}^{-} / S^{\prime}}\left(S_{1, i}^{\prime}+\cdots+S_{m_{i}, i}^{\prime}\right)\right)^{\otimes n} \hookrightarrow \mathbb{P}\left(f_{S^{\prime}}\right)_{*}\left(\omega_{\mathcal{C}_{S^{\prime}} / S^{\prime}}\right)^{\otimes n}$, where we may assume that $n$ has been chosen large enough a priori. Put $N_{i}^{-}:=\left(2 g_{i}^{-}-\right.$ $\left.2+m_{i}\right) n-g_{i}^{-}+1$. There is a principal PGL $\left(N_{i}^{-}+1\right)$-bundle $p_{i}^{-}: E_{i}^{-} \rightarrow S^{\prime}$ associated to the projective bundle $\mathbb{P}\left(f_{S^{\prime}, i}^{-}\right)_{*}\left(\omega_{\mathcal{C}_{S^{\prime}, i}^{-} / S^{\prime}}\left(S_{1, i}^{\prime}+\cdots+S_{m_{i}, i}^{\prime}\right)\right)^{\otimes n}$.

This bundle is naturally a subbundle of the principal PGL $(N+1)$-bundle $p_{S^{\prime}}: E_{S^{\prime}} \rightarrow S^{\prime}$ associated to the projective bundle $\mathbb{P}\left(f_{S^{\prime}}\right)_{*}\left(\omega_{\mathcal{C}_{S^{\prime}} / S^{\prime}}\right)^{\otimes n}$, which is equal to the pullback of the principal bundle $p: E \rightarrow S$ from above. Indeed, there is a natural inclusion of $\mathbb{P}\left(f_{S^{\prime}, i}^{-}\right)_{*}\left(\omega_{\mathcal{C}_{S^{\prime}, i}^{-} / S^{\prime}}\left(S_{1, i}^{\prime}+\cdots+\right.\right.$ $\left.\left.S_{m_{i}, i}^{\prime}\right)\right)^{\otimes n}$ into $\mathbb{P}\left(f_{S^{\prime}}\right)_{*}\left(\omega_{\mathcal{C}_{S^{\prime}} / S^{\prime}}\right)^{\otimes n}$, compare 4.4 . Viewing $A$ as a subgroup of $\operatorname{PGL}\left(N_{i}^{-}+1\right)$, this induces an embedding of the respective quotients with respect to the action of $A$, and therefore an inclusion $E_{i}^{-} / A \hookrightarrow E_{S^{\prime}} / A$. Recall from 3.1 that $A$ is a normal subgroup of $\operatorname{Aut}\left(C_{0}^{-} ; \mathcal{Q}\right)$, with quotient $\operatorname{Aut}\left(C_{0}^{-} ; \mathcal{Q}\right) / A=\Gamma$. Since the embedding of the projective bundles is invariant with respect to reorderings of the labels of the marked points, i.e. with respect to the action of $\Gamma$, there is thus even an induced embedding

$$
E_{i}^{-} / \operatorname{Aut}\left(C_{0} ; \mathcal{Q}\right) \longleftrightarrow E_{S^{\prime}} / A
$$


For $i=1, \ldots, r$ we define $f_{E_{i}}^{-}: \mathcal{C}_{E_{i}}^{-} \rightarrow E_{i}^{-}$as the pullback of the stable curve $f_{S^{\prime}, i}^{-}: \mathcal{C}_{S^{\prime}, i}^{-} \rightarrow S^{\prime}$ along $p_{i}^{-}$. Each is an $m_{i}$-pointed stable curve of genus $g_{i}^{-}$over $E_{i}^{-}$, with sections of marked points $\tilde{S}_{1, i}^{\prime}, \ldots, \tilde{S}_{m_{i}, i}^{\prime}$. The projective bundle $\mathbb{P}\left(f_{E_{i}}^{-}\right)_{*}\left(\omega_{\mathcal{C}_{E_{i}}^{-} / E_{i}}\left(\tilde{S}_{1, i}^{\prime}+\cdots+\tilde{S}_{m_{i}, i}^{\prime}\right)\right)^{\otimes n}$ has a natural trivialization, compare also Remark 5.6. Hence by the universal property of the Hilbert scheme there is an induced morphism $\vartheta_{i}^{-}: E_{i}^{-} \rightarrow \bar{H}_{g_{i}^{-}, n, m_{i}}$. which is $\operatorname{PGL}\left(N_{i}^{-}+1\right)$-equivariant.

(ii) Now we want to put these constructions on the connected components $\mathcal{C}_{S^{\prime}, i}^{-}$together for the whole prestable curve $f_{S^{\prime}}^{-}: \mathcal{C}_{S^{\prime}}^{-} \rightarrow S^{\prime}$. We define $P^{-}:=\operatorname{PGL}\left(N_{1}^{-}+1\right) \times \cdots \times \operatorname{PGL}\left(N_{r}^{-}+1\right)$, and $\bar{H}_{g^{-}, n, m}^{-}:=\bar{H}_{g_{1}^{-}, n, m_{1}} \times \cdots \times$ $\bar{H}_{g_{r}^{-}, n, m_{r}}$. We obtain a principal $P^{-}$-bundle $p_{S^{\prime}}^{-}: E_{S^{\prime}}^{-}:=E_{i}^{-} \times \cdots \times E_{r}^{-} \rightarrow S^{\prime}$, together with an induced $P^{-}$-equivariant morphism $\vartheta_{S^{\prime}}^{-}: E_{S^{\prime}}^{-} \rightarrow \bar{H}_{g^{-}, n, m}^{-}$.

By construction, the induced morphism $\mathcal{C}_{E_{1}}^{-} \times \cdots \times \mathcal{C}_{E_{r}}^{-} / P^{-} \rightarrow E_{S^{\prime}}^{-} / P^{-}$ on the quotients is just the $m$-pointed prestable curve $f_{S^{\prime}}^{-}: \mathcal{C}_{S^{\prime}}^{-} \rightarrow S^{\prime}$ of genus $g^{-}$over $S^{\prime}$. Hence for the induced morphism $\vartheta^{\prime}: S^{\prime} \rightarrow \bar{M}_{g^{-}, m}^{-}$holds $\vartheta^{\prime} \circ p_{S^{\prime}}^{-}=\pi \circ \vartheta_{S^{\prime}}^{-}$, where $\pi: \bar{H}_{g^{-}, n, m}^{-} \rightarrow \bar{M}_{g^{-}, m}^{-}:=\bar{M}_{g_{1}^{-}, m_{1}} \times \cdots \times \bar{M}_{g_{r}^{-}, m_{r}}$ denotes the canonical quotient morphism. Note that by construction all fibres of $f_{S^{\prime}}^{-}: \mathcal{C}_{S^{\prime}}^{-} \rightarrow S^{\prime}$ are isomorphic to $C_{0}^{-}$, hence $\vartheta^{\prime}$ is constant. So there is in fact a commutative diagram

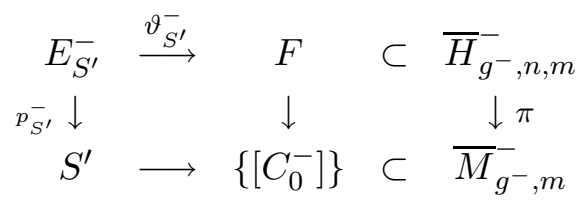

where $F \cong P^{-} / A$ is the fibre of the canonical morphism $\bar{H}_{g^{-}, n, m}^{-} \rightarrow \bar{M}_{g^{-}, m}^{-}$ over the point $\left[C_{0}^{-}\right]$representing the isomorphism class of $C_{0}^{-}$.

Fix an embedding of $C_{0}^{-}$into $\mathbb{P}^{N_{1}^{-}} \times \cdots \times \mathbb{P}^{N_{r}^{-}}$, corresponding to a point in $\bar{H}_{g^{-}, n, m}^{-}$, which shall also be denoted by $\left[C_{0}^{-}\right]$. Note that the decomposition of $A=\operatorname{Aut}\left(C_{0}^{-}\right)$into a product $\operatorname{Aut}\left(C_{1}^{-}\right) \times \cdots \times \operatorname{Aut}\left(C_{r}^{-}\right)$is compatible with the decomposition of $P^{-}$, since each connected component of $C_{0}^{-}$contains at least one marked point. A standard computation now shows that

$$
E_{S^{\prime}}^{-} / A \cong F \times S^{\prime}
$$

is a trivial fibration. Indeed, we can define a trivializing section $\sigma^{\prime}: S^{\prime} \rightarrow$ $E_{S^{\prime}}^{-} / A$ as follows. For $s \in S^{\prime}$ choose some $e \in\left(p_{S^{\prime}}^{-}\right)^{-1}(s) \subset E_{S^{\prime}}^{-}$, such that 
$\vartheta_{S^{\prime}}^{-}(e)=\left[C_{0}^{-}\right] \in F \subset \bar{H}_{g^{-}, n, m}^{-}$. Under the identification $F \cong P^{-} / A$ the point $\left[C_{0}^{-}\right]$corresponds to the class [id] of the identity id $\in P^{-}$. Hence by the $P^{-}$-equivariance of $\vartheta_{S^{\prime}}^{-}$, the point $e$ is uniquely defined up to the action of $A$, and thus $\sigma^{\prime}(s):=[e] \in E_{S^{\prime}}^{-} / A$ is well defined.

(iii) So far we have been working on an étale cover $u: S^{\prime} \rightarrow S$. From the construction of the section $\sigma^{\prime}: S^{\prime} \rightarrow E_{S^{\prime}}^{-} / A$ one sees that, if $s_{1}, s_{2} \in S^{\prime}$ are closed points such that $u\left(s_{1}\right)=u\left(s_{2}\right)$, then $\sigma^{\prime}\left(s_{1}\right)$ and $\sigma^{\prime}\left(s_{2}\right)$ differ at most by an element in $\operatorname{Aut}\left(C_{0}^{-} ; \mathcal{Q}\right) / A=\Gamma$. Thus we can define a section $\bar{\sigma}$ : $S \rightarrow E_{S^{\prime}}^{-} / \operatorname{Aut}\left(C_{0}^{-} ; \mathcal{Q}\right)$. Using the inclusion $E_{S^{\prime}}^{-} / \operatorname{Aut}\left(C_{0} ; \mathcal{Q}\right) \hookrightarrow E_{S^{\prime}} / A$, and composing it with the canonical quotient morphism, as well as the pullback morphism $E_{S^{\prime}} \rightarrow E$, we finally obtain a section $\sigma: S \rightarrow E / P \times A$. We now define a subbundle $E^{+}$of the principal PGL $(N+1)$-bundle $p: E \rightarrow S$ by the following Cartesian diagram:

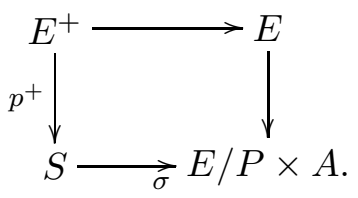

It is easy to see that $p^{+}: E^{+} \rightarrow S$ is a principal $P \times A$-bundle, and its extension $\left(E^{+} \times \operatorname{PGL}(N+1)\right) /(P \times A)$ is isomorphic to $E$. Note that this property makes this construction the inverse of the construction of Proposition 6.6.

(iv) Recall that our aim is to construct an object $\left(E^{+}, p^{+}, \phi^{+}\right) \in$ $\left[H_{g^{+}, n, m / \Gamma}^{\times} / P \times A\right](S)$. To do this, we stil need to define an $P \times A$-equivariant morphism $\phi^{+}: E^{+} \rightarrow H_{g^{+}, n, m / \Gamma}^{\times}$.

Let $v: K^{\prime} \rightarrow K^{\times}$be an étale cover as in Lemma 5.4, such that the pullback $f_{K^{\prime}}: \mathcal{C}_{K^{\prime}} \rightarrow K^{\prime}$ of the universal curve $\mathcal{C}_{g, n, 0} \rightarrow K^{\times}$contains a trivial subcurve $f_{K^{\prime}}^{-}: C_{0}^{-} \times K^{\prime} \rightarrow K^{\prime}$. Let $f_{K^{\prime}}^{+}: \mathcal{C}_{K^{\prime}}^{+} \rightarrow K^{\prime}$ denote the closure of the complement of this trivial subcurve. This is an $m$-pointed stable curve of genus $g^{+}$over $K^{\prime}$. By construction, $E^{+} \subset E$ is a subbundle, which is stabilized under the action of the subgroup $P$ in $\operatorname{PGL}(N+1)$. Thus the $\operatorname{PGL}(N+1)$-equivariant morphism $\phi: E \rightarrow K^{\times}$restricts to a $P$-equivariant morphism $\phi_{K}^{+}: E^{+} \rightarrow K^{\times}$.

Consider the pullback $\phi_{v}^{+}: E_{v}^{+}:=v^{*} E^{+} \rightarrow K^{\prime}$ of this morphism along $v$. We define an $m$-pointed stable curve $f_{v}^{+}: \mathcal{C}_{v}^{+} \rightarrow E_{v}^{+}$of genus $g^{+}$as the pullback of the stable curve $f_{K^{\prime}}^{+}: \mathcal{C}_{K^{\prime}}^{+} \rightarrow K^{\prime}$ along $\phi_{v}^{+}$. The projective bundle $\mathbb{P}\left(f_{v}^{+}\right)_{*}\left(\omega_{\mathcal{C}_{v}^{+} / E_{v}^{+}}\left(S_{1}+\cdots+S_{m}\right)\right)^{\otimes n}$ on $E_{v}^{+}$is isomorphic to the pullback of 
the projective bundle $\mathbb{P}\left(f_{K^{\prime}}^{+}\right)_{*}\left(\omega_{\mathcal{C}_{K^{\prime}}^{+} / K^{\prime}}\left(\mathcal{S}_{1}+\cdots+\mathcal{S}_{m}\right)\right)^{\otimes n}$ on $K^{\prime}$ along the morphism $\phi_{v}^{+}$. Here again $S_{1}, \ldots, S_{m}$ and $\mathcal{S}_{1}, \ldots, \mathcal{S}_{m}$ denote the divisors of the $m$ marked points on $\mathcal{C}_{v}^{+}$and $\mathcal{C}_{K^{\prime}}^{+}$, respectively.

$(v)$ Note that there is a distinguished trivialization of the projective bundle $\mathbb{P}\left(f_{K^{\prime}}^{+}\right)_{*}\left(\omega_{\mathcal{C}_{K^{\prime}}^{+} / K^{\prime}}\left(\mathcal{S}_{1}+\cdots+\mathcal{S}_{m}\right)\right)^{\otimes n}$ over $K^{\prime}$. This can be seen as follows. The curve $f_{K^{\prime}}: \mathcal{C}_{K^{\prime}} \rightarrow K^{\prime}$ has been constructed as the pullback of the universal curve on $\bar{H}_{g, n, 0}$, restricted to $K^{\times}$. Therefore there exists a natural embedding of the subcurve $f_{K^{\prime}}^{+}: \mathcal{C}_{K^{\prime}}^{+} \rightarrow K^{\prime}$ into $\mathbb{P}^{N} \times K^{\prime} \rightarrow K^{\prime}$. To distinguish a trivialization of $\mathbb{P}\left(f_{K^{\prime}}^{+}\right)_{*}\left(\omega_{\mathcal{C}_{K^{\prime}}^{+} / K^{\prime}}\left(\mathcal{S}_{1}+\cdots+\mathcal{S}_{m}\right)\right)^{\otimes n}$ over $K^{\prime}$, it suffices to distinguish a corresponding embedding of the subcurve $f_{K^{\prime}}^{+}: \mathcal{C}_{K^{\prime}}^{+} \rightarrow K^{\prime}$ into $\mathbb{P}^{N^{+}} \times K^{\prime} \rightarrow K^{\prime}$.

Consider first the curve $f^{\times}: \mathcal{C}_{g^{+}, n, m}^{\times} \rightarrow H_{g^{+}, n, m}^{\times}$, which is the restriction of the universal curve on the full Hilbert scheme. By definition, this curve is embedded into $\mathbb{P}^{N^{+}} \times H_{g^{+}, n, m}^{\times}=\mathbb{P} f_{*}^{\times}\left(\omega_{\mathcal{C}_{g^{+}, n, m}^{\times}} / H_{g^{+}, n, m}^{\times}\left(\mathcal{S}_{1}+\cdots+\right.\right.$ $\left.\left.\mathcal{S}_{m}\right)\right)^{\otimes n}$, which in turn is embedded into $\mathbb{P}\left(u_{0}\right)_{*}\left(\omega_{\mathcal{C}_{0} / H_{g^{+}, n, m}^{\times}}\right)^{\otimes n}=\mathbb{P}^{N} \times$ $H_{g^{+}, n, m}^{\times}$by the natural inclusion of $\mathbb{P} f_{*}^{\times}\left(\omega_{\mathcal{C}_{g^{+}, n, m}^{\times}} / H_{g^{+}, n, m}^{\times}\left(\mathcal{S}_{1}+\ldots+\mathcal{S}_{m}\right)\right)^{\otimes n}$ in $\mathbb{P}\left(u_{0}\right)_{*}\left(\omega_{\mathcal{C}_{0} / H_{g^{+}, n, m}^{\times}}\right)^{\otimes n}$. Compare construction 4.4 for details and for the notation. Note that the embedding is fibrewise independent of the ordering of the labels of the marked points. We used this in Proposition 4.11 for the construction of the morphism $\Theta^{\times}: H_{g^{+}, n, m}^{\times} \rightarrow \bar{H}_{g, n, 0}$. Recall that $\Theta^{\times}$ induces a morphism $\Theta: H_{g^{+}, n, m / \Gamma}^{\times} \rightarrow \bar{H}_{g, n, 0}$, see Proposition 4.16.

For each closed point $[C] \in \Theta\left(H_{g^{+}, n, m / \Gamma}^{\times}\right)=\Theta^{\times}\left(H_{g^{+}, n, m}^{\times}\right)$representing a stable curve $C$ embedded into $\mathbb{P}^{N}$, there is a unique subcurve isomorphic to $C_{0}^{-}$, and thus a unique subcurve $C^{+}$, which is an $m / \Gamma$-pointed stable curve of genus $g^{+}$, satisfying $\Theta\left(\left[C^{+}\right]\right)=[C]$. Thus, the restriction of $f_{K^{\prime}}^{+}: \mathcal{C}_{K^{\prime}}^{+} \rightarrow K^{\prime}$ to the preimage $T^{\prime}$ of $\Theta\left(H_{g^{+}, n, m / \Gamma}^{\times}\right)$has a distinguished embedding into $\mathbb{P}^{N^{+}} \times T^{\prime} \rightarrow T^{\prime}$, namely the one given by the embedding of the universal curve over $H_{g^{+}, n, m}^{\times}$. We want to extend this embedding to all of $K^{\prime}$, using Lemma 5.2, which implies that $K^{\times}=\Theta\left(H_{g^{+}, n, m / \Gamma}^{\times}\right) \cdot \operatorname{PGL}(N+1)$. Note that if $x, y \in K^{\prime}$ are closed points with $v(x)=v(y)$, then the fibres of $f_{K^{\prime}}^{+}$ over the two points differ only by a reordering of the labels of their marked points, which does not affect their embedding into $\mathbb{P}^{N^{+}}$.

Let $[C] \in T$ be a closed point representing a stable curve $C$ embedded into $\mathbb{P}^{N}$, with a decomposition $C=C^{+} \cup C_{0}^{-}$as above, together with the embedding of $C^{+}$into $\mathbb{P}^{N^{+}}$. For an element $\gamma \in \operatorname{PGL}(N+1)$, the distinguished 
$m / \Gamma$-pointed stable subcurve of genus $g^{+}$of the translated embedded curve $\gamma C$ is the curve $\gamma C^{+}$, by the uniqueness statement of Corollary 5.5. The obvious embedding of $\gamma C^{+}$into $\gamma \mathbb{P}^{N^{+}} \subset \mathbb{P}^{N}$ is in fact independent of the choice of $\gamma$.

Indeed, let $\gamma^{\prime} \in \operatorname{PGL}(N+1)$ be another element such that $\gamma^{\prime}([C])=$ $\gamma([C])$. Then $\gamma^{\prime} \circ \gamma^{-1} \in \operatorname{Aut}(C)$. By the definition of $\mathcal{D}^{\times}\left(C_{0} ; P_{1}\right)$, this group splits as $\operatorname{Aut}(C)=\operatorname{Aut}_{\Gamma}\left(C^{+} ; \mathcal{Q}\right) \times A$. Since the embedding of $C^{+}$ into $\mathbb{P}^{N^{+}}$is independent of the ordering of the labels of the marked points, and since $\operatorname{Aut}\left(C^{+}\right)=\operatorname{Stab}_{\mathrm{PGL}\left(N^{+}+1\right)}\left(\left[C^{+}\right]\right)$, any elements of $\operatorname{Aut}(C)$ lying in $\operatorname{Aut}_{\Gamma}\left(C^{+} ; \mathcal{Q}\right)$ leave the embedding invariant. By construction 4.6, there is a fixed embedding $C_{0}^{-} \subset \mathbb{P}^{N^{-}} \subset \mathbb{P}^{N}$, and thus inclusions $A \subset \operatorname{PGL}\left(N^{-}+1\right) \subset$ $\operatorname{PGL}(N+1)$. Note that $\operatorname{PGL}\left(N^{-}+1\right)$ acts trivially on $\mathbb{P}^{N^{+}}$outside the intersection $\mathbb{P}^{N^{+}} \cap \mathbb{P}^{N^{-}}$. However, elements of $A$ fix by definition the marked points of $C_{0}^{-}$, which span the intersection $\mathbb{P}^{N^{+}} \cap \mathbb{P}^{N^{-}}$as a subspace of $\mathbb{P}^{N}$, and thus fix the subspace $\mathbb{P}^{N^{+}}$. Hence elements of Aut $(C)$ lying in $A$ fix the embedding of $C^{+}$as well.

Therefore, for all closed points $[C] \in K^{\times}$, representing an embedded stable curve $C \subset \mathbb{P}^{N}$, there is a well-defined embedding of the distinguished $m / \Gamma$-pointed stable subcurve $C^{+} \subset C$ into $\mathbb{P}^{N^{+}}$, and so there exists an embedding of $f_{K^{\prime}}^{+}: \mathcal{C}_{K^{\prime}}^{+} \rightarrow K^{\prime}$ into $\mathbb{P}^{N^{+}} \times K^{\prime} \rightarrow K^{\prime}$, as claimed.

(vi) So finally we obtain an $m$-pointed stable curve $f_{v}^{+}: \mathcal{C}_{v}^{+} \rightarrow E_{v}^{+}$, together with a trivialization of the projective bundle $\mathbb{P}\left(f_{v}^{+}\right)_{*}\left(\omega_{\mathcal{C}_{v}^{+} / E_{v}^{+}}\left(S_{1}+\right.\right.$ $\left.\left.\cdots+S_{m}\right)\right)^{\otimes n}$. By the universal property of the Hilbert scheme, this defines a $P$-equivariant morphism $\tilde{\phi}_{v}^{+}: E_{v}^{+} \rightarrow H_{g^{+}, n, m}^{\times}$.

Recall that $E_{v}^{+}=v^{*} E^{+}$. If $e_{1}, e_{2} \in E$ get mapped to the same point in $E^{+}$, then by the construction of $\tilde{\phi}_{v}^{+}$there exists a permutation $\gamma \in \Gamma$, such that $\gamma \tilde{\phi}_{v}^{+}\left(e_{1}\right)=\tilde{\phi}_{v}^{+}\left(e_{2}\right)$. Therefore there is a well-defined induced morphism

$$
\phi^{+}: E^{+} \longrightarrow H_{g^{+}, n, m / \Gamma}^{\times},
$$

which is $P \times A$-equivariant, with $A$ acting trivially on $H_{g^{+}, n, m / \Gamma}^{\times}$.

(vii) Taking everything together, we obtain an object $\left(E^{+}, p^{+}, \phi^{+}\right)$of the fibre category $\left[H_{g^{+}, n, m / \Gamma}^{\times} / P \times A\right](S)$. Note that up to the action of $A$ this triple just represents the $m / \Gamma$-pointed stable curve over $S$, which is the closure of the complement of the étale trivial curve $C_{0}^{-} \times S \rightarrow S$ in $f_{S}: \mathcal{C}_{S} \rightarrow S$, as in Lemma 5.4 .

$\mathrm{Up}$ to isomorphism, this construction of the reduction $E^{+}$of $E$ is inverse to the extension used in the proof of Proposition 6.6. In fact one 
has the equality $\Xi\left(\Lambda\left(E^{+}, p^{+}, \phi^{+}\right)\right)=\left(E^{+}, p^{+}, \phi^{+}\right)$, and an isomorphism $\Lambda(\Xi(E, p, \phi)) \cong(E, p, \phi)$.

(viii) It finally remains to define the inverse functor $\Xi$ for all schemes $S$ on morphisms $\varphi:\left(E_{2}, p_{2}, \phi_{2}\right) \rightarrow\left(E_{1}, p_{1}, \phi_{1}\right)$ in $\mathcal{D}^{\times}\left(C_{0} ; P_{1}\right)(S)$. Such a morphism is given by a morphism of principal $\operatorname{PGL}(N+1)$-bundles $f$ : $E_{1} \rightarrow E_{2}$, such that the diagram

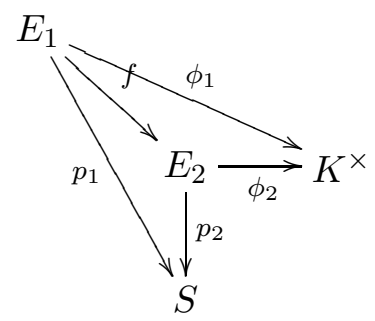

commutes. We need to show that $f$ restricts to a morphism of principal $P \times A$-bundles $f^{+}: E_{1}^{+} \rightarrow E_{2}^{+}$, where $E_{1}^{+}$and $E_{2}^{+}$are the $P \times A$-subbundles of $E_{1}$ and $E_{2}$ constructed as above. Note that this restriction, if it exists, necessarily satisfies $\phi_{1}=\phi_{2} \circ f^{+}$.

For $i=1,2$, the morphism $\phi_{i}$ factors as $\phi_{i}=\Theta \circ \phi_{i}^{+}$, where $\Xi\left(E_{i}, p_{i}, \phi_{i}\right)$ $=\left(E_{i}^{+}, p_{i}^{+}, \phi_{i}^{+}\right)$. The injectivity of $\Theta: H_{g^{+}, n, m / \Gamma}^{\times} \rightarrow K^{\times}$, which follows from Proposition 4.16, implies the identity $\phi_{1}^{+}=\phi_{2}^{+} \circ f^{+}$, so $f^{+}: E_{1}^{+} \rightarrow E_{2}^{+}$defines indeed a morphism between the triples $\left(E_{2}^{+}, p_{2}^{+}, \phi_{2}^{+}\right)$and $\left(E_{1}^{+}, p_{1}^{+}, \phi_{1}^{+}\right)$. It is clear from the construction that this inverts the definition of $\Lambda$ on morphisms. Thus $\Xi$ is shown to be an inverse functor to $\Lambda$, up to isomorphism, provided that we can prove the existence of $f^{+}$.

Clearly, such a restriction $f^{+}: E_{1}^{+} \rightarrow E_{2}^{+}$exists, if and only if the condition $f\left(E_{1}^{+}\right) \subseteq E_{2}^{+}$is satisfied.

To do this, let us briefly recall our construction of $\Xi$ on objects. Let $i=1,2$. We used the morphism $\phi_{i}: E_{i} \rightarrow K^{\times}$to pull back the universal curve $\mathcal{C}_{g, n, 0} \rightarrow K^{\times}$to $E_{i}$. This pullback contains a distinguished $m$-pointed prestable curve $f_{E_{i}}^{-}: \mathcal{C}_{E_{i}}^{-} \rightarrow E_{i}$ as a subcurve, which in turn induces a morphism $\vartheta_{i}^{-}: E_{i}^{-} \rightarrow \bar{H}_{g^{-}, n, m}^{-}$. Strictly speaking, this is true only up to étale coverings. However, the above inclusion condition is not affected by étale pullbacks, so we may safely ignore this technical complication.

By the universal property of the Hilbert space, we have the identity

$$
\vartheta_{1}^{-}=\vartheta_{2}^{-} \circ f
$$


We saw that $\vartheta_{i}^{-}$is in fact a morphism $\vartheta_{i}^{-}: E_{i}^{-} \rightarrow F$, where $F \cong P^{-} / A$ is the fibre of the quotient morphism $\bar{H}_{g^{-}, n, m}^{-} \rightarrow \bar{M}_{g^{-}, m}^{-}$over the point representing the isomorphism class of $C_{0}^{-}$. Again, we identify here the image [id] of the unit id $\in P^{-} / A$ with the point $\left[C_{0}^{-}\right]$representing the fixed embedding of $C_{0}^{-}$.

There is a section $\sigma_{i}^{\prime}: S \rightarrow E_{i}^{-} / A$, sending a point $s \in S$ to the equivalence class of points $e \in E_{i}^{-}$in the fibre over $s$ with $\vartheta_{i}^{-}(e)=[\mathrm{id}] \in$ $F$. This is well-defined because $\vartheta_{i}^{-}$is $P^{-}$-equivariant. Let $\sigma_{i}$ denote the morphism from $S$ to $E_{i} / P \times A$ induced by $\sigma_{i}^{\prime}$. Now the bundle $E_{i}^{+}$is defined as the unique subbundle of $E_{i}$, which fits into the Cartesian diagram

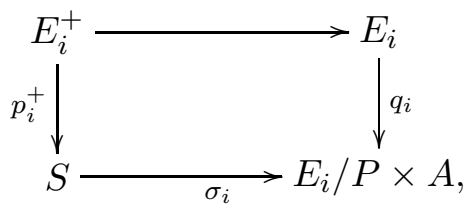

where $q_{i}: E_{i} \rightarrow E_{i} / P \times A$ denotes the natural quotient map. In other words, for an element $e \in E_{i}$ holds $e \in E_{i}^{+}$if and only if $q_{i}(e)=\sigma_{i}\left(p_{i}^{+}(e)\right)$, which in turn is equivalent to $\vartheta_{i}^{-}(e)=[\mathrm{id}]$ by the definition of $\sigma_{i}$. So the relation $f\left(E_{1}^{+}\right) \subseteq E_{2}^{+}$translates into the condition $\vartheta_{2}^{-}(f(e))=[\mathrm{id}]$ for all $e \in E_{1}^{+}$. However, above we found the identity $(*) \vartheta_{1}^{-}=\vartheta_{2}^{-} \circ f$, which concludes the proof.

Corollary 6.10. An isomorphism of stacks

$$
\mathcal{D}^{\times}\left(C_{0} ; P_{1}\right) \cong \mathcal{M}_{g^{+}, n, m / \Gamma\left(C_{0}^{-} ; \mathcal{Q}\right)}^{\times}
$$

exists if and only if $\operatorname{Aut}\left(C_{0}^{-}\right)$is trivial.

Proof. This follows immediately from Theorem 6.8 .

COROLlary 6.11. There exists a natural morphism of moduli stacks

$$
\Omega: \mathcal{M}_{g^{+}, n, m / \Gamma\left(C_{0}^{-} ; \mathcal{Q}\right)}^{\times} \longrightarrow \mathcal{D}^{\times}\left(C_{0} ; P_{1}\right)
$$

which is representable, surjective, finite and unramified, and of degree equal to the order of the group $\operatorname{Aut}\left(C_{0}^{-}\right)$. 
Proof. Using the notation from the proof of Theorem 6.8, the morphism $\Omega$ is given as the composition of the canonical quotient morphism $\left[H_{g^{+}, n, m / \Gamma}^{\times} / P\right] \rightarrow\left[H_{g^{+}, n, m / \Gamma}^{\times} / P \times A\right]$ with the isomorphism $\Lambda$ from Proposition 6.6. Note that the quotient morphism is a representable, finite and unramified surjective morphism. Since both $\mathcal{M}_{g^{+}, n, m / \Gamma\left(C_{0}^{-} ; \mathcal{Q}\right)}^{\times}$and $\mathcal{D}^{\times}\left(C_{0} ; P_{1}\right)$ are smooth stacks of dimension one by Lemma 6.3 , this implies that $\Omega$ is étale as well.

Remark 6.12. Summarizing the above results, we obtain the following commutative diagram:

$$
\begin{array}{cccccc}
H_{g^{+}, n, m / \Gamma}^{\times} & = & H_{g^{+}, n, m / \Gamma}^{\times} & \hookrightarrow & \bar{H}_{g, n, 0} \\
\mathcal{M}_{g^{+}, m / \Gamma} & & & \mathcal{D}^{\times}\left(C_{0} ; P_{1}\right) & \hookrightarrow & \overline{\mathcal{M}}_{g} \\
\downarrow & & \downarrow & & \downarrow \\
M_{g^{+}, m / \Gamma}^{\times} & \cong & D^{\times}\left(C_{0} ; P_{1}\right) & \hookrightarrow & \bar{M}_{g} .
\end{array}
$$

Note that, since $\operatorname{Aut}\left(C_{0}^{-}\right)$acts trivially, there is also a canonical morphism

$$
\mathcal{D}^{\times}\left(C_{0} ; P_{1}\right) \cong\left[H_{g^{+}, n, m / \Gamma}^{\times} / P \times A\right] \longrightarrow\left[H_{g^{+}, n, m / \Gamma}^{\times} / P\right] \cong \mathcal{M}_{g^{+}, m / \Gamma}^{\times},
$$

which is of degree equal to $\frac{1}{\# \operatorname{Aut}\left(C_{0}^{-}\right)}$. In [Z2] it is shown that the moduli stack of $m / \Gamma$-pointed stable curves is a quotient of the moduli stack of $m$-pointed stable curves with respect to the action of $\Gamma$. This implies in particular that there is a finite surjective morphisms

$$
\bar{\Omega}: \mathcal{M}_{g^{+}, m}^{\times} \longrightarrow \mathcal{D}^{\times}\left(C_{0} ; P_{1}\right),
$$

which is of degree equal to the order of $\operatorname{Aut}\left(C_{0}^{-} ; \mathcal{Q}\right)$. In general, this morphism is not representable, see [Z1].

\section{$\S 7$. The case of genus three}

To illustrate the description of one-dimensional boundary substacks of the moduli space of Deligne-Mumford stable curves as given above, we want to consider the case of genus $g=3$ in detail.

Remark 7.1. ( $i$ ) There are exactly five different stable curves of genus $g=3$ with $3 g-3=6$ nodes. We denote them by $C_{1}, \ldots, C_{5}$, in the same order as they are listed in Faber's paper $[\mathrm{F}]$. Schematically they can be represented as in the following pictures. 
THE ONE-DIMENSIONAL STRATUM IN THE BOUNDARY OF $\overline{\mathcal{M}}_{g}$
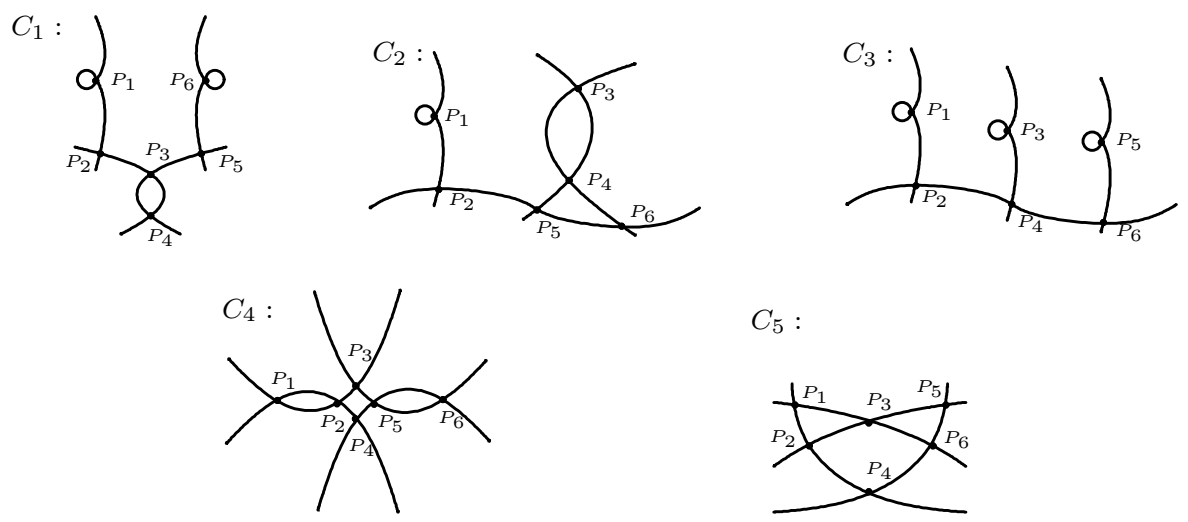

$C_{5}$ :

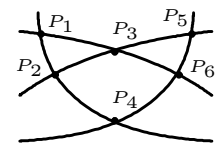

(ii) There are eight different types of stable curves of genus 3 with exactly 5 nodes. Following Faber's notation, we denote them by $(a), \ldots,(h)$. They are represented by the pictures below.
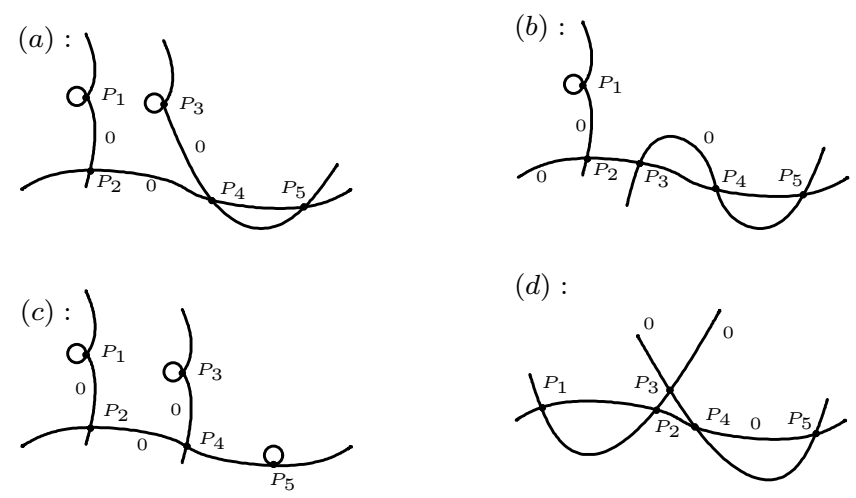

$(d):$
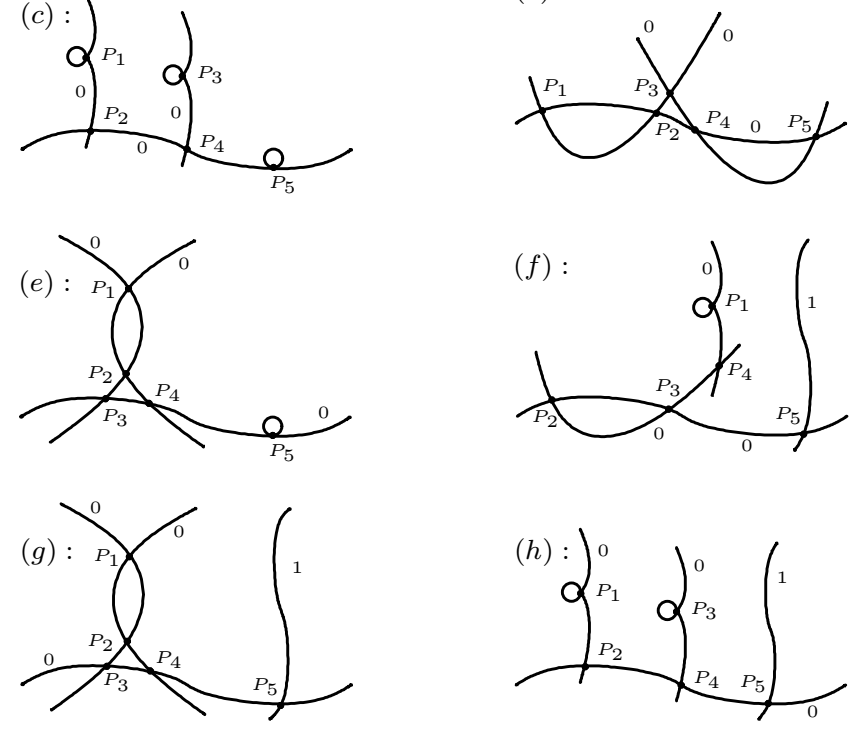

The number next to an irreducible component gives its geometric genus. 
Remark 7.2. The type of a curve containing $3 g-4=5$ nodes does not vary within a connected component $D$ of the boundary stratum $M_{3}^{(5)}$. Recall that each such component can be written as $D=D\left(C_{i} ; P_{j}\right)$ for some $1 \leq i \leq 5$ and $1 \leq j \leq 6$. The following table lists all possible presentations for all connected components.

\begin{tabular}{lllccc}
$D$ & $D\left(C_{i} ; P_{j}\right)$ & $M_{g^{+}, m}^{\prime}$ & $\operatorname{deg}(\bar{\Omega})$ & $\operatorname{Aut}\left(C_{i}^{-}\right)$ & $\Gamma\left(C_{i}^{-} ; \mathcal{Q}\right)$ \\
\hline & & & & & \\
$(a)$ & $D\left(C_{1} ; P_{2,5}\right)$ & $M_{1,2}^{\prime}$ & 4 & $\mathbb{Z}_{2}$ & $\Sigma_{2}$ \\
& $D\left(C_{2} ; P_{3,4}\right)$ & $M_{1,2}^{\prime}$ & 4 & $\mathbb{Z}_{2}$ & $\Sigma_{2}$ \\
$(b)$ & $D\left(C_{2} ; P_{5,6}\right)$ & $M_{0,4}$ & 12 & $\mathbb{Z}_{2}$ & $\Sigma_{3}$ \\
$(c)$ & $D\left(C_{1} ; P_{3,4}\right)$ & $M_{1,2}^{\prime}$ & 8 & $\mathbb{Z}_{2} \times \mathbb{Z}_{2}$ & $\Sigma_{2}$ \\
& $D\left(C_{3} ; P_{2,4,6}\right)$ & $M_{1,2}^{\prime}$ & 8 & $\mathbb{Z}_{2} \times \mathbb{Z}_{2}$ & $\Sigma_{2}$ \\
$(d)$ & $D\left(C_{4} ; P_{3,4}\right)$ & $M_{0,4}$ & 8 & $\mathrm{id}$ & $D_{8}$ \\
& $D\left(C_{5} ; P_{1, \ldots, 6}\right)$ & $M_{0,4}$ & 8 & $\mathrm{id}$ & $D_{8}$ \\
$(e)$ & $D\left(C_{2} ; P_{2}\right)$ & $M_{1,2}^{\prime}$ & 4 & $\mathbb{Z}_{2}$ & $\Sigma_{2}$ \\
& $D\left(C_{4} ; P_{1,2,5,6}\right)$ & $M_{1,2}^{\prime}$ & 4 & $\mathbb{Z}_{2}$ & $\Sigma_{2}$ \\
$(f)$ & $D\left(C_{1}: P_{1,6}\right)$ & $M_{1,1}$ & 4 & $\mathbb{Z}_{2} \times \mathbb{Z}_{2}$ & id \\
$(g)$ & $D\left(C_{2} ; P_{1}\right)$ & $M_{1,1}$ & 4 & $\mathbb{Z}_{2} \times \mathbb{Z}_{2}$ & id \\
$(h)$ & $D\left(C_{3} ; P_{1,3,5}\right)$ & $M_{1,1}$ & 8 & $D_{8}$ & id
\end{tabular}

The first column specifies the connected component $D$ by indicating the type of a general point. The second column lists all possible choices of curves $C_{i}$ and nodes $P_{j}$ to represent the respective component. More than one subscript, like for example $P_{2,5}$, indicates that both nodes $P_{2}$ and $P_{5}$ produce the same component $D$. The third column gives the moduli scheme $M_{g^{+}, m}^{\prime}$ covering $D$ as in Lemma 3.4, and the fourth column states the degree of the finite morphism $\bar{\Omega}: \mathcal{M}_{g^{+}, m}^{\times} \rightarrow \mathcal{D}^{\times}\left(C_{i} ; P_{j}\right)$ as in Remark 6.12. Columns five and six give the corresponding group of automorphisms $\operatorname{Aut}\left(C_{i}^{-}\right)$and the subgroup of permutations $\Gamma\left(C_{i}^{-} ; \mathcal{Q}\right) \subset \Sigma_{m}$, respectively. Here, $D_{8}$ denotes the dihedral group of order 8 .

Example 7.3. Consider the stable curve $C_{2}$. There are two pairs of equivalent nodes on $C_{2}$, in the sense that for each pair there exists an automorphism of $C_{2}$ interchanging the two nodes. Therefore deformations, which preserve all but one node of $C_{2}$, distinguish four different connected components $D$ of $M_{3}^{(5)}$. Let us have a closer look at all four of those components. In particular, we want to apply the isomorphism from Theorem 
6.8 to the corresponding moduli substacks $\mathcal{D}$. We quote from [Z1] to supply additional information about possible extensions of this isomorphism.

(i) We consider first the connected component $D=D\left(C_{2} ; P_{1}\right)$. A general point of this component represents a curve of type $(g)$. Since $m=1$, all automorphism groups split. In particular, we have the equality $D^{\times}\left(C_{2} ; P_{1}\right)=D\left(C_{2} ; P_{1}\right)$. Thus we have an isomorphism $\mathcal{D}\left(C_{2} ; P_{1}\right) \cong$ $\mathcal{M}_{1,1} / \mathbb{Z}_{2} \times \mathbb{Z}_{2}$ by Theorem 6.8 . In fact, in this case the isomorphism extends to the respective closures $\overline{\mathcal{D}}\left(C_{2} ; P_{1}\right) \cong \overline{\mathcal{M}}_{1,1} / \mathbb{Z}_{2} \times \mathbb{Z}_{2}$.

(ii) Consider now $D=D\left(C_{2} ; P_{2}\right)$, where a general point of this component represents a curve of type $(e)$. Note that for all points $\left[C^{+}\right] \in$ $M_{1,2}^{\prime}$, representing a stable curve $C^{+}$with two marked points, the automorphism group $\operatorname{Aut}(C)$ splits. Thus there is an isomorphism $\mathcal{D}\left(C_{2} ; P_{2}\right) \cong$ $\mathcal{M}_{1,2 / \Sigma_{2}}^{\prime} / \mathbb{Z}_{2}$. It can be extended to a partial compactification of $\mathcal{D}^{\times}\left(C_{2} ; P_{2}\right)$ over the point $\left[C_{2}\right]$, but not to its closure.

(iii) The case of $D=D\left(C_{2} ; P_{3}\right)$ is analogous to case $(i i)$. Note that this is the same connected component as $D\left(C_{2} ; P_{4}\right)$, where a general point represents a curve of type $(a)$. There is also an isomorphism $\mathcal{D}\left(C_{2} ; P_{3}\right) \cong$ $\mathcal{M}_{1,2 / \Sigma_{2}}^{\prime} / \mathbb{Z}_{2}$, which can be extended to a partial compactification, but not to all of the closure.

(iv) Finally, consider the connected component $D=D\left(C_{2} ; P_{5}\right)=$ $D\left(C_{2} ; P_{6}\right)$, with general point of type $(b)$. Again we have an equality $D^{\times}\left(C_{2} ; P_{5}\right)=D\left(C_{2} ; P_{5}\right)$. There is an isomorphism of stacks $\mathcal{D}\left(C_{2} ; P_{5}\right) \cong$ $\mathcal{M}_{0,4 / \Sigma_{3}} / \mathbb{Z}_{2}$. This isomorphism cannot be extended any further. However, one can find a natural reduced substack $\hat{\mathcal{M}}_{2,1}$ of the moduli stack $\overline{\mathcal{M}}_{2,1}$ of 1-pointed stable curves of genus 2 , so that for the closure of $\mathcal{D}\left(C_{2} ; P_{5}\right)$ there is an isomorphism $\overline{\mathcal{D}}\left(C_{2} ; P_{5}\right) \cong \hat{\mathcal{M}}_{2,1} / \mathbb{Z}_{2}$. For full details, see [Z1].

EXAMPLE 7.4. To provide an example, where the isomorphism of Theorem 6.8 does not hold for all of $D$, look at the connected component $D=$ $D\left(C_{3} ; P_{2}\right)$. A general point represents a curve of type $(c)$. If $\left[C^{+}\right] \in M_{1,2}^{\prime}$ represents a stable curve $C^{+}$with two marked points, which are in a special position, then the exact sequence of automorphism groups of Lemma 3.2 does not split. There is an isomorphism $\mathcal{D}^{\times}\left(C_{3} ; P_{2}\right) \cong \mathcal{M}_{1,2 / \Sigma_{2}}^{\times} / \mathbb{Z}_{2} \times \mathbb{Z}_{2}$, but it cannot be extended any further. 


\section{REFERENCES}

[DM] P. Deligne and D. Mumford, The irreducibility of the space of curves of a given genus, Publ. Math. I.H.E.S., 36 (1969), 75-109.

[E] D. Edidin, Notes on the construction of the moduli space of curves, Recent progress in intersection theory (G. Ellingsrud, et al., eds.), 2000, pp. 85-113.

[F] C. Faber, Chow rings of moduli spaces of curves $I$ : The Chow ring of $\overline{\mathcal{M}}_{3}$, Annals of Maths., 132 (1990), 331-419.

[G] D. Gieseker, Lectures on moduli of curves, Tata Institute Lecture Notes 69, 1982.

[HM] J. Harris and I. Morrison, Moduli of Curves, Springer-Verlag, New York, 1998.

[K] F. Knudsen, The projectivity of the moduli space of stable curves, II: the stacks $M_{g, n}$, Math. Scand., 52 (1983), 161-199.

[Z1] J. Zintl, One-dimensional substacks of the moduli stack of Deligne-Mumford stable curves, Habilitationsschrift, Kaiserslautern, 2005, math. AG/0612802.

[Z2] J. Zintl, Moduli stacks of permutation classes of pointed stable curves, Milan j. math., 76 (2008), 401-418, math.AG/0611710.

Fachbereich Mathematik

Technische Universität Kaiserslautern

Postfach 3049

67653 Kaiserslautern

Germany

zintl@mathematik.uni-kl.de 